\title{
Quasimaps and stable pairs
}

\author{
Henry Liu \\ Department of Mathematics, Columbia University, 2990 Broadway, New York NY 10027, USA; E-mail: \\ hliu@math.columbia.edu.
}

Received: 20 August 2020; Revised: 5 December 2020; Accepted: 3 March 2021

2020 Mathematics Subject Classification: Primary - 14N35; Secondary - 14C05

\begin{abstract}
We prove an equivalence between the Bryan-Steinberg theory of $\pi$-stable pairs on $Y=\mathcal{A}_{m-1} \times \mathbb{C}$ and the theory of quasimaps to $X=\operatorname{Hilb}\left(\mathcal{A}_{m-1}\right)$, in the form of an equality of K-theoretic equivariant vertices. In particular, the combinatorics of both vertices are described explicitly via box counting. Then we apply the equivalence to study the implications for sheaf-counting theories on $Y$ arising from 3D mirror symmetry for quasimaps to $X$, including the Donaldson-Thomas crepant resolution conjecture.
\end{abstract}

\section{Introduction}

\subsection{Curve counting}

\subsection{1.}

Given a smooth variety $Z$, one can construct many compactifications of the moduli space of smooth curves in $Z$. These moduli spaces differ in how they treat the data of a curve in $Z$.

- Viewing a curve as the data of a map $f: C \rightarrow Z$ and allowing the domain $C$ to develop nodal singularities in the compactification yields moduli spaces of stable maps; for example, as in Gromov-Witten (GW) theory.

- Viewing a curve as the data of an ideal sheaf $\mathcal{I}_{C} \subset \mathcal{O}_{Z}$ and allowing $C$ to degenerate into 1dimensional subschemes in the compactification yields moduli spaces of sheaves; for example, as in Donaldson-Thomas (DT) theory.

Morally, one expects all enumerative theories of curves in $Z$ to be equivalent, possibly up to some wallcrossing behaviour: a change of variables, analytic continuation and/or normalisation. One example of such an equivalence is the celebrated GW/DT correspondence [27] [28], proved for all toric 3-folds in [29]. Consequently, tools from DT-like sheaf-counting theories can be applied with great effectiveness to GW theory and vice versa; for example, the proof of the Igusa cusp form conjecture in [35].

\subsection{2.}

The main result of this article, Theorem 35, is another (much simpler) such equivalence, at the level of equivariant and $K$-theoretic curve counts. For a moduli space $\mathcal{M}$, working in equivariant K-theory means that the enumerative invariant to be considered is not the cohomological partition function

$$
Z_{\mathcal{M}}^{\text {coh }}:=\sum_{\text {deg }} x^{\operatorname{deg}} \int_{\left[\mathcal{M}_{\text {deg }}\right]} 1 \in H_{\mathrm{T}}^{*}(\mathrm{pt})_{\text {localised }}\left[\left[\boldsymbol{x}^{ \pm}\right]\right]
$$

(C) The Author(s), 2021. Published by Cambridge University Press. This is an Open Access article, distributed under the terms of the Creative Commons Attribution licence (http://creativecommons.org/licenses/by/4.0/), which permits unrestricted re-use, distribution, and reproduction in any medium, provided the original work is properly cited 
but rather the $K$-theoretic partition function

$$
\mathrm{Z}_{\mathcal{M}}:=\sum_{\operatorname{deg}} \boldsymbol{x}^{\mathrm{deg}} \chi\left(\mathcal{M}_{\mathrm{deg}}, \hat{\mathcal{O}}_{\mathcal{M}}^{\mathrm{vir}}\right) \in K_{\mathrm{T}}(\mathrm{pt})_{\text {localised }}\left[\left[\boldsymbol{x}^{ \pm}\right]\right]
$$

Here, $\boldsymbol{x}$ are variables recording the degree of the curve along with any other discrete data parametrising connected components of $\mathcal{M}=\bigsqcup_{\text {deg }} \mathcal{M}_{\text {deg }}$. The virtual structure sheaf $\mathcal{O}_{\mathcal{M}}^{\text {vir }}$ (see, e.g., [25, Section 2.3], [8, Section 3.2]) is the K-theoretic analogue of the virtual fundamental class $[\mathcal{M}]^{\text {vir }}$. Its symmetrised version $\hat{\mathcal{O}}_{\mathcal{M}}^{\text {vir }}$ is roughly $\mathcal{O}_{\mathcal{M}}^{\text {vir }} \otimes \mathcal{K}_{\text {vir }}^{1 / 2}$ where $\mathcal{K}_{\text {vir }}:=\operatorname{det}\left(T^{\text {vir }}\right)^{\vee}$ is the virtual canonical. The importance of the twist by $\mathcal{K}_{\mathrm{vir}}^{1 / 2}$ is discussed in [36, Section 3.2].

K-theoretic invariants recover cohomological invariants in a particular limit and are therefore richer and more general. Equivariance gives us a handle on partition functions via certain quantum differential/difference equations that they satisfy. These features make equivariant K-theory a productive setting for enumerative geometry; see [36] for an introduction.

\subsection{3.}

In this article we study only the genus- 0 setting $g(C)=0$, where $C=\mathbb{P}^{1}$ when $C$ is smooth. Degeneration arguments reduce a given general $C$ to this case.

\subsection{The enumerative theories}

\subsection{1.}

In the case where the target space $X=V / /{ }_{\theta} G$ is a sufficiently nice geometric invariant theory (GIT) quotient, the theory of stable quasimaps [9] provides an alternate compactification for maps $f: \mathbb{P}^{1} \rightarrow X$ that is more amenable to computation, among other nice properties, compared to stable maps. It is related to GW theory via a series of wall-crossings whose composition gives the classical mirror map of [16] [26]. Roughly, a quasimap to $V / /{ }_{\theta} G$ is a choice of principal $G$-bundle $\mathcal{P}$ on $\mathbb{P}^{1}$ and a section $s \in H^{0}\left(\mathbb{P}^{1}, \mathcal{P} \times_{G} V\right)$ satisfying a stability condition. See Subsection 3.3 for details.

Quasimap theory is especially rich when $X$ is a particularly nice class of GIT quotients called Nakajima quiver varieties [31]. These are smooth symplectic varieties associated to quivers and are closely related to moduli of quiver representations, moduli of sheaves on symplectic surfaces and, from physics, moduli of vacua in $3 \mathrm{D} \mathcal{N}=4$ supersymmetric gauge theories. Hence, their curve counts often have deep representation-theoretic or physical meaning.

\subsection{2.}

Let $Q \operatorname{Maps}(X)$ be the moduli of quasimaps to a Nakajima quiver variety $X$. One can ask whether there is some kind of sheaf-counting theory on some space related to $X$ whose partition function is equal to $Z_{\text {QMaps }}(X)$. Indeed, if $X=\operatorname{Hilb}(S)$ is the Hilbert scheme of points on a surface $S$, the graph construction gives a rough equivalence

$$
\left(f: \mathbb{P}^{1} \rightarrow \operatorname{Hilb}(S)\right) \approx\left(\begin{array}{c}
1 \text {-dimensional subscheme of } S \times \mathbb{P}^{1} \\
\text { with nonzero degree along } \mathbb{P}^{1}
\end{array}\right)
$$

The simplest Nakajima quiver varieties of the form $\operatorname{Hilb}(S)$ are when $S$ is an ADE surface, namely, a minimal resolution of $\mathbb{C}^{2} / \Gamma$ for a finite subgroup $\Gamma \subset \operatorname{SL}(2, \mathbb{C})$. So, from now on, let $S$ be an ADE surface. Then $\operatorname{Hilb}(S)$ is the Nakajima quiver variety associated to the affine ADE quiver corresponding to $S$. 


\subsection{3.}

Let $Y:=S \times \mathbb{P}^{1}$. To match with quasimaps to $X=\operatorname{Hilb}(S)$, DT theory is not the correct sheaf-counting theory to take on $Y$. DT theory counts 1-dimensional subschemes $C \subset Y$ and therefore its moduli space is

$$
\mathrm{DT}(Y):=\operatorname{Hilb}(Y, \text { curves }) .
$$

But arbitrary 1-dimensional subschemes include two types of unwanted components that do not occur for quasimaps:

1. 0-dimensional components that range freely over $Y$;

2. 1-dimensional components that lie purely in a fibre $S \times\{\mathrm{pt}\} \subset Y$.

\subsection{4.}

One way to remove the unwanted contributions of type 1 is to take the DT partition function $Z_{D T}$ and divide by the partition function for the moduli

$$
\mathrm{DT}_{0}(Y):=\operatorname{Hilb}(Y, \text { points })
$$

of points on $Y$. A better and more geometric way is to use Pandharipande-Thomas (PT) theory [40]. Roughly, if DT theory counts surjections

$$
\mathcal{O}_{Y} \stackrel{s}{\rightarrow} \mathcal{O}_{C} \rightarrow 0
$$

PT theory counts stable pairs $\left[\mathcal{O}_{Y} \rightarrow \mathcal{F}\right]$ in

$$
\mathcal{O}_{Y} \stackrel{s}{\rightarrow} \mathcal{F} \rightarrow \mathcal{Q} \rightarrow 0
$$

where $\mathcal{Q}$ is 0 -dimensional. We think of this as allowing the map $s$ in DT theory to develop a 0-dimensional cokernel $\mathcal{Q}$ instead of being a surjection.

If Pairs $(Y)$ denotes the moduli of stable pairs, the well-known conjectural DT/PT correspondence predicts that $^{1}$

$$
Z_{\text {Pairs }}(Y)=\frac{Z_{\mathrm{DT}}(Y)}{Z_{\mathrm{DT}_{0}}(Y)} \text {. }
$$

\subsection{5.}

To remove the unwanted contributions of type 2, we can repeat the DT/PT story as follows. A fibre of $Y \rightarrow \mathbb{P}^{1}$ is an ADE surface $S$, so it has an exceptional divisor $E$ for the resolution $\pi: S \rightarrow \mathbb{C}^{2} / \Gamma$. Let Pairs $\operatorname{exc}(Y)$ be the moduli of stable pairs on $Y$ supported only on $E$ for some fibre. For our purposes, $Z_{\text {Pairs }_{\text {exc }}}$ is the correct series to normalise by.

Bryan-Steinberg (BS) theory [5] provides a geometric approach to this normalisation. Roughly, whereas PT theory allows the cokernel $\mathcal{Q}$ to be 0 -dimensional, BS theory allows $\mathcal{Q}$ to develop 1-dimensional components supported only on $E$. Such pairs $\left[\mathcal{O}_{Y} \stackrel{s}{\rightarrow} \mathcal{F}\right]$ are called $\pi$-stable pairs. A precise definition is in Subsection 2.2.1. Because it depends not only on $Y$ but also on the resolution $\pi$, we denote the BS moduli space $\pi$-Pairs $(Y)$.

As with the DT/PT correspondence, the conjectural PT/BS correspondence is that ${ }^{2}$

$$
Z_{\pi \text {-Pairs }}(Y)=\frac{Z_{\text {Pairs }}(Y)}{Z_{\text {Pairsexc }}(Y)}
$$

\footnotetext{
${ }^{1}$ Here is one place to emphasise the importance of using the symmetrised $\hat{\mathcal{O}}^{\text {vir }}$ instead of $\mathcal{O}^{\text {vir }}$ : the DT/PT correspondence (and probably similar wall-crossings) fails to hold if we just use $\mathcal{O}^{\text {vir }}$.

${ }^{2}$ As with the DT/PT correspondence, at the K-theoretic level this also requires the symmetrised $\hat{\mathcal{O}}^{\text {vir }}$.
} 
Assuming the DT/PT correspondence, one can replace the right-hand side by $Z_{\mathrm{DT}}(X) / \mathrm{Z}_{\mathrm{DT}}$ exc $(X)$ where $\mathrm{DT}_{\text {exc }}$ is defined in exactly the same way as Pairs $\mathrm{exc}_{\text {. }}$.

\subsection{Main result(s)}

\subsection{1.}

The main result of this article is the BS/quasimaps correspondence of Theorem 35, which implies that

$$
\mathrm{Z}_{\pi \text {-Pairs }}\left(\mathcal{A}_{m-1} \times \mathbb{P}^{1}\right)=\mathrm{Z}_{\mathrm{QMaps}}\left(\operatorname{Hilb}\left(\mathcal{A}_{m-1}\right)\right)
$$

but is a more precise statement. Namely, for toric geometries, $\mathbf{Z}$ factors as a product of contributions from torus-fixed points and torus-invariant curves. These contributions are called vertices $\vee$ and edges $\mathrm{E}$, respectively, and one can match vertices and edges for different enumerative theories individually. Though it is very straightforward to match edges for DT/PT/BS and quasimaps, vertices have a certain combinatorial complexity. The more precise content of Theorem 35 is that

$$
\mathrm{V}_{\pi \text {-Pairs }}\left(\mathcal{A}_{m-1} \times \mathbb{C}\right)=\mathrm{V}_{\mathrm{QMaps}}\left(\operatorname{Hilb}\left(\mathcal{A}_{m-1}\right)\right) .
$$

Both (2) and (3) should also be refined in this way, using the DT [27] and PT [39] vertices.

For 3-folds, DT/PT vertices are objects on $\mathbb{C}^{3}$ and have three legs, corresponding to what happens along noncompact torus-fixed curves. Legs are where vertices glue onto edges. In BS theory, the notion of a vertex depends on the resolution $\pi$. For $Y=S \times \mathbb{P}^{1}$, the BS vertex is an object on $S \times \mathbb{C}$. It has one set of $m$ legs from the $\mathbb{C}$ direction and two legs from $S$. We say the BS vertex has one leg when these latter two legs are empty. Then $Z_{\pi \text {-Pairs }}\left(S \times \mathbb{P}^{1}\right)$ is the gluing of two BS 1-leg vertices along an edge. In this language, (4) says the BS 1-leg vertex is equal to the quasimap vertex. Note that in quasimap theory there is no notion of 2-leg or 3-leg vertices.

\subsection{2.}

In Subsection 2.1, we set up notation for $Y=\mathcal{A}_{m-1} \times \mathbb{C}$ and its torus action, and then in Subsection 2.2 we define the BS vertex. The proof of the BS/quasimaps correspondence goes via equivariant localisation, and so Subsection 2.3 gives an explicit combinatorial description of the BS 1-leg vertex as a weighted sum over certain 3D box configurations similar to 3D partitions. An important detail, discussed in Subsection 2.4, is that even for the 1-leg vertex the moduli of $\pi$-stable pairs has torus-fixed loci of arbitrarily large dimension; such a phenomenon is not present in DT or PT theory.

In Subsection 3.1, we begin by understanding $\operatorname{Hilb}\left(\mathcal{A}_{m-1}\right)$ and a related space $\operatorname{Hilb}\left(\left[\mathbb{C}^{2} / \Gamma\right]\right)$ as Nakajima quiver varieties. Here $\left[\mathbb{C}^{2} / \Gamma\right]$ is the CY3 orbifold associated to $\mathcal{A}_{m-1}$. Though we will not need $\operatorname{Hilb}\left(\left[\mathbb{C}^{2} / \Gamma\right]\right)$ for the BS/quasimaps correspondence, it plays a significant role later in the DT crepant resolution conjecture. Subsection 3.2 gives a combinatorial description of the torus-equivariant geometry of both Hilbert schemes using 2D box configurations similar to Young diagrams. Then Subsection 3.3 defines the quasimap vertex and explicitly describes it as a weighted sum over such labelled $2 \mathrm{D}$ box configurations.

\subsection{3.}

The statement and proof of the BS/quasimap correspondence are presented in Section 4. Although we prove it only for the geometry $\mathcal{A}_{m-1} \times \mathbb{P}^{1}$ for ease of exposition, the correspondence certainly extends beyond the type A case to any ADE bundle over $\mathbb{P}^{1}$. This is the most general setting in which comparable BS and quasimap theories can be defined. For type D and type E, the combinatorial interpretation of contributions to the quasimap vertex in terms of coloured boxes is less straightforward.

The proof of the BS/quasimaps correspondence involves constructing an isomorphism of torus-fixed loci that respects the tangent-obstruction theories. This is done using the torus-equivariant derived McKay equivalence of Subsection 4.2. That it matches the stability conditions defining $\pi$-stable pairs and quasimaps is the content of Subsection 4.3, where we also show that the isomorphism of fixed 
loci extends to an isomorphism of BS and quasimap 1-leg moduli spaces, thereby showing that the two theories are truly equivalent.

\subsection{4.}

The theory of quasimaps to Nakajima quiver varieties can be used to study $3 D$ mirror symmetry, also known as symplectic duality [19]. This is an intimate (conjectural) relationship between two mirror Nakajima quiver varieties $X$ and $\check{X}$ that relates their quasimap vertices; see Subsection 5.1 for more details. The relationship between the two quasimap vertices, once pushed through the BS/quasimaps correspondence, yields a remarkable range of known results; for example, the geometric engineering of certain Nekrasov partition functions (Subsection 5.2), and the DT crepant resolution conjecture (Subsection 5.3). In particular, these results are very special limits of the full 3D mirror symmetry, which yields a (conjectural) statement of the DT crepant resolution conjecture (CRC) for equivariant K-theoretic vertices.

\section{Bryan-Steinberg pairs}

\subsection{The threefold}

2.1.1.

Though many constructions in this article can be done more generally, the basic geometry of interest is

$$
Y:=\mathcal{A}_{m-1} \times C
$$

for a curve $C$ that will either be $\mathbb{C}$ or $\mathbb{P}^{1}$. The surface $S:=\mathcal{A}_{m-1}$ is the minimal resolution of the type $\mathrm{A}$ singularity $\mathbb{C}^{2} / \Gamma$. Here, $\mathbb{C}^{2}$ has the canonical symplectic form, and

$$
\Gamma:=\mathbb{Z} / m \subset \operatorname{SL}(2, \mathbb{C})
$$

acts by the symplectomorphism

$$
\xi \cdot(x, y):=\left(\xi x, \xi^{-1} y\right) .
$$

By an abuse of notation, we conflate the coordinate functions $(x, y, z)$ on $\mathbb{C}^{2} \times C$ with the weights of the torus

$$
\mathrm{T}:=\mathbb{C}_{x}^{\times} \times \mathbb{C}_{y}^{\times} \times \mathbb{C}_{z}^{\times}
$$

acting on it. ${ }^{3}$ Then the minimal resolution $\mathcal{A}_{m-1}$ has exceptional divisor consisting of a chain of $m-1$ copies of $\mathbb{P}^{1}$, which we denote $E_{1}, \ldots, E_{m-1}$, with weights as depicted in Figure 1 .

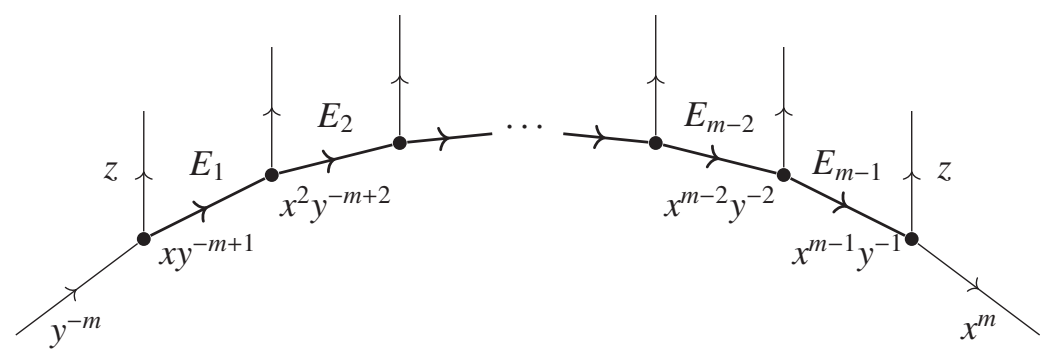

Figure 1. Toric diagram of $\mathcal{A}_{m-1} \times \mathbb{C}$ (exceptional divisor of $\mathcal{A}_{m-1}$ in bold).

\footnotetext{
${ }^{3}$ Our convention for weights is opposite to some parts of the literature, notably [36] (which develops K-theoretic quasimap theory) and successive works. For example, if $\mathbb{C}_{z}^{\times}$acts on $\mathbb{A}^{1}$, then for us the character of $\mathcal{O}_{\mathbb{A}^{1}}$ is $1 /(1-z)$, whereas for [36] it would be $1 /\left(1-z^{-1}\right)$. On the other hand, our convention does agree with older works such as [27] (which develops DT theory).
} 
Let $p_{0}, \ldots, p_{m}$ denote the T-fixed points, such that $E_{a}$ connects $p_{a-1}$ and $p_{a}$. Let

$$
\begin{aligned}
& x_{a}:=x^{a+1} y^{-m+a+1} \\
& y_{a}:=x^{-a} y^{m-a}
\end{aligned}
$$

so that $\left(x_{a}, y_{a}, z\right)$ are coordinates for the toric chart $U_{a}=\mathbb{C}^{3}$ around $p_{a}$.

\subsection{2.}

Often, it will be necessary to switch between working on $Y$ and working on $S$. To prevent confusion, let

$$
\mathrm{T}^{\prime}:=\mathbb{C}_{x}^{\times} \times \mathbb{C}_{y}^{\times} \subset \mathrm{T}
$$

be the torus acting on the $\mathbb{C}^{2}$ defining $S$. In general, we will add a prime to any equivariant object when considering it on $S$ instead of $Y$. For example, let

$$
\mathrm{A}:=\{x y z=1\} \subset \mathrm{T}
$$

be the Calabi-Yau subtorus. Then

$$
\mathrm{A}^{\prime}=\left\{\left(t, t^{-1}\right)\right\} \subset \mathrm{T}^{\prime}=\mathrm{A}^{\prime} \times \mathbb{C}_{\hbar}^{\times},
$$

where we use $\hbar:=1 / x y$ to denote the weight of the symplectic form on $S$. Then $\mathrm{A}^{\prime}$ preserves the symplectic form on $S$ and contains the $\Gamma$-action defining $S$.

\subsection{The 1-leg vertex}

\subsection{1.}

Very generally, let $Y_{0}$ be a quasi-projective 3-fold with rational Gorenstein singularities and let $\pi: Y \rightarrow Y_{0}$ be a resolution of singularities of relative dimension $\leq 1$. Let $\operatorname{Coh}_{\leq i}(Y) \subset \operatorname{Coh}(Y)$ denote the full subcategory of coherent sheaves with support in dimension $\leq i$. Associated to $\pi$ is a torsion pair $(\mathrm{T}, \mathrm{F})$ in $\mathrm{Coh}_{\leq 1}(Y)$, given by

$$
\begin{aligned}
& \mathrm{T}:=\left\{\mathcal{Q} \in \mathrm{Coh}_{\leq 1}(Y) \mid R \pi_{*} \mathcal{Q} \in \mathrm{Coh}_{\leq 0}\left(Y_{0}\right)\right\} \\
& \mathrm{F}:=\left\{\mathcal{F} \in \mathrm{Coh}_{\leq 1}(Y) \mid \operatorname{Hom}(\mathcal{Q}, \mathcal{F})=0 \text { for all } \mathcal{Q} \in \mathrm{T}\right\}=\mathrm{T}^{\perp}
\end{aligned}
$$

A BS pair [5] for $\pi$, also called a $\pi$-stable pair, is a map

$$
s: \mathcal{O}_{Y} \rightarrow \mathcal{F}
$$

such that $\mathcal{F} \in \mathrm{F}$ and $\operatorname{coker}(s) \in \mathrm{T}$. Equivalently, a $\pi$-stable pair is a short exact sequence

$$
0 \rightarrow \mathcal{O}_{C} \rightarrow \mathcal{F} \rightarrow \mathcal{Q} \rightarrow 0
$$

where $\mathcal{O}_{C}:=\operatorname{im}(s)$ and $\mathcal{Q}:=\operatorname{coker}(s)$. We know $\operatorname{im}(s)$ is the structure sheaf of some curve $C$ because it is a quotient of $\mathcal{O}_{Y}$ supported in dimension 1.

Remark 1. Due to (1), we only want to consider 3-folds $Y$ of the form $S \times \mathbb{P}^{1}$, where the surface $S$ contains the rational Gorenstein singularities. For surfaces it is known that the only such singularities are of ADE type [21, Theorem 7.5.1]. 


\subsection{2.}

Let $\pi$-Pairs $(Y)$ denote the moduli of $\pi$-stable pairs on $Y$. When $Y=S \times \mathbb{P}^{1}$, let $D:=S \times\{\infty\}$ be the divisor at infinity and consider the open locus

$$
\pi \text {-Pairs }(Y)_{\text {nonsing } \infty} \subset \pi \text {-Pairs }(Y)
$$

where the evaluation map

$$
\begin{aligned}
\operatorname{ev}_{\infty}: \pi \text {-Pairs }(Y)_{\text {nonsing } \infty} & \rightarrow \operatorname{Hilb}(D, \text { points }) \\
{\left[\mathcal{O}_{Y} \rightarrow \mathcal{F}\right] } & \left.\mapsto \mathcal{F}\right|_{\infty}
\end{aligned}
$$

lands in the Hilbert scheme of points on $D$, instead of the Hilbert scheme of curves.

Definition 2. The BS 1-leg vertex is the series

$$
\mathrm{V}_{\pi \text {-Pairs }}(Q, \boldsymbol{A}):=\sum_{\substack{n \in \mathbb{Z} \\ \beta \in H_{2}(S, \mathbb{Z})}} Q^{n} A^{\beta} \mathrm{ev}_{\infty, *}\left(\pi \text {-Pairs }^{n, \beta}, \hat{\mathcal{O}}^{\mathrm{vir}}\right) \in K_{\mathrm{T}}(S)_{\text {localised }}((Q))[[\boldsymbol{A}]]
$$

The variables $Q$ and $\boldsymbol{A}:=\left(A_{1}, \ldots, A_{m-1}\right)$ record certain discrete data indexing the connected components $\pi$-Pairs ${ }^{n, \beta} \subset \pi$-Pairs $(Y)_{\text {nonsing } \infty}$ :

○ the box-counting variable $Q$ records the (possibly negative) renormalised volume $n:=$ $\chi_{\text {normalised }}(\mathcal{F})$

$\circ$ the Kähler variables $\boldsymbol{A}$ record the degree $\beta:=\operatorname{deg}(\mathcal{F})$ along components $E_{1}, \ldots, E_{m-1}$ of the exceptional divisor.

This is all the same as in DT or PT theory; see [27, Section 4.4] for details.

\subsection{3.}

The moduli $\pi$-Pairs $(Y)$ inherits the T-action on $Y$. For $p \in \operatorname{Hilb}(S)$, let

$$
\pi \text {-Pairs }{ }_{p}:=\operatorname{ev}_{\infty}^{-1}(p)
$$

It has components $\pi$-Pairs ${ }_{p}^{n, \beta}$ consisting of $\pi$-stable pairs with $\chi_{\text {normalised }}=n$ and deg $=\beta$. Then the T-fixed locus decomposes as

$$
\pi \text {-Pairs }(Y)_{\text {nonsing } \infty}^{\mathrm{T}}=\bigoplus_{p \in \operatorname{Hilb}(S)^{\mathrm{T}^{\prime}}} \bigoplus_{\beta \in H_{2}(S, \mathbb{Z})} \bigoplus_{n \in \mathbb{Z}}\left(\pi \text {-Pairs }{ }_{p}^{n, \beta}\right)^{\mathrm{T}},
$$

and the $p$ th component of the BS 1-leg vertex is therefore

$$
\mathrm{V}_{\pi \text {-Pairs }}^{p}(Q, \boldsymbol{A})=\sum_{\beta \in H_{2}(S, \mathbb{Z})} \sum_{n \in \mathbb{Z}} \chi\left(\pi \text {-Pairs }{ }_{p}^{n, \beta}, \hat{\mathcal{O}}^{\text {vir }}\right) Q^{n} A^{\beta}
$$

In Subsection 2.4, we roughly describe the fixed loci $\left(\pi-\mathrm{Pairs}_{p}^{n, \beta}\right)^{\mathrm{T}}$ and argue that they are proper. Hence, the BS 1-leg vertex is well defined via localisation. Note that for a given $\beta$, the renormalised volume $n$ is bounded from below, and so the coefficients of the power series in $\boldsymbol{A}$ are Laurent series in $Q$.

\subsection{4.}

The appropriate tangent-obstruction theory for a $\pi$-stable pair arises from viewing it as a two-term complex

$$
I^{\bullet}:=\left[\mathcal{O}_{Y} \rightarrow \mathcal{F}\right] \in D^{b} \operatorname{Coh}(Y),
$$


as for stable pairs [40, Section 2]. Then there is a universal formula

$$
\begin{aligned}
T_{\left[I^{\bullet}\right]}^{\mathrm{vir}} & =\operatorname{Ext}^{1}\left(I^{\bullet}, I^{\bullet}\right)_{0}-\operatorname{Ext}^{2}\left(I^{\bullet}, I^{\bullet}\right)_{0} \\
& =\chi\left(\mathcal{O}_{Y}\right)-\chi\left(I^{\bullet}, I^{\bullet}\right)
\end{aligned}
$$

for the virtual tangent space. Here $\chi(-,-):=\sum_{i}(-1)^{i} \operatorname{Ext}^{i}(-,-)$. As in DT or PT theory, at fixed points $\left[I^{\bullet}\right] \in(\pi \text {-Pairs })^{\mathrm{T}}$ the T-character of $T^{\text {vir }}$ can be obtained via Čech cohomology. The computation is identical in DT/PT/BS theory and we will not repeat it here.

Usually for the vertex of a DT-like theory, one redistributes vertex and edge contributions so that the 1-leg vertex $\mathrm{V}^{p}$ does not include the contribution of the infinite leg(s) (see [27, Section 4.9]). We have not performed such a redistribution in (7); in our setup, for $I^{\bullet} \in \pi$-Pairs ${ }_{\text {nonsing } \infty}^{T}$, the redistribution says to use

$$
T_{\left[I^{\bullet}\right], \sim}^{\mathrm{vir}}:=T_{\left[I^{\bullet}\right]}^{\mathrm{vir}}-T_{\mathrm{ev}_{\infty}\left(I^{\bullet}\right)} \operatorname{Hilb}(S)
$$

instead of $T_{\left[I^{\bullet}\right]}^{\mathrm{vir}}$. However, this redistribution is essentially responsible for the renormalisation of the Euler characteristic $n=\chi$ normalised $(\mathcal{F})$, and we $d o$ perform this renormalisation.

\subsection{Boxes and rods}

\subsection{1.}

Because the geometry $Y=\mathcal{A}_{m-1} \times \mathbb{P}^{1}$ is toric, a T-fixed $\pi$-stable pair $\left[\mathcal{O}_{Y} \rightarrow \mathcal{F}\right]$ can be described by toric data. Namely, $\mathcal{F}$ can be described as a configuration of boxes in each toric chart $U_{a} \subset Y$. This is the combinatorial approach taken in DT and PT theory as well; see [27, Section 4.2] and [39, Section 2] for details.

Our convention for box diagrams is as follows. All box diagrams will be drawn on the toric skeleton of Figure 1. A box drawn with smallest $x$-, $y$ - and $z$-coordinates $(i, j, k)$ in the chart $U_{a}=\mathbb{C}^{3}$ indicates that the $\mathbb{C}\left[x_{a}, y_{a}, z\right]$-module $\mathcal{F}\left(U_{a}\right)$ has an element of weight 四 $=x_{a}^{i} y_{a}^{j} z^{k}$. (We often conflate the coordinates $(i, j, k)$ of a box with its weight $x_{a}^{i} y_{a}^{j} z^{k}$.) Importantly, it is possible for $\mathcal{F}\left(U_{a}\right)$ to contain an $m$-dimensional vector subspace of weight 四, where $m>1$. In this case, we label the box with the integer $m$, which we think of as a multiplicity. ${ }^{4}$

\subsection{2.}

Elements $\mathcal{F} \in \pi$-Pairs $(Y)_{\text {nonsing } \infty}^{\mathrm{T}}$ correspond to box configurations on the geometry $\mathcal{A}_{m-1} \times \mathbb{C}$ with infinite legs along the $z$ direction, as in Figure 2. These legs are described by an $m$-tuple of partitions

$$
\lambda=\left(\lambda_{0}, \ldots, \lambda_{m-1}\right)
$$

corresponding to a $\mathrm{T}^{\prime}$-fixed point in $\operatorname{Hilb}\left(\mathcal{A}_{m-1}\right)$. More precisely, the infinite leg in the chart $U_{a}$ is the module

$$
L_{a}:=\mathbb{C}\left[x_{a}, y_{a}, z\right] / I_{\lambda_{a}}
$$

where $I_{\lambda_{a}}$ is the ideal generated by $x_{a}^{i(\square)} y_{a}^{j(\square)}$ for every $\square \notin \lambda_{a}$.

Among the T-invariant curves in $Y$, the noncompact (respectively compact) ones are called external (respectively internal) legs in box configurations. In addition to the legs $L_{a}$ of weight $z$, there are two other external legs with weights $x^{m}$ and $y^{m}$. In principle, one can set up the theory so that these external legs can be nonempty as well. The resulting vertices would be the BS 2-leg or 3-leg vertices. In our setting they must be empty, and hence we call the resulting series the 1-leg vertex.

${ }^{4}$ This phenomenon is not new to BS theory; the PT 3-leg vertex is allowed to have certain boxes of multiplicity 2, and this phenomenon is important there because it leads to positive-dimensional T-fixed loci. 


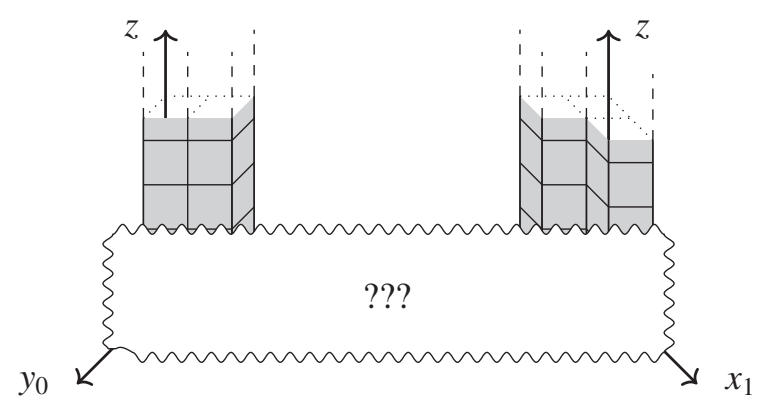

Figure 2. An element of $\pi$-Pairs $(2),(2,1)\left(\mathcal{A}_{1}\right)_{\text {nonsing } \infty}$.

\subsection{3.}

The overarching goal is to characterise the valid box configurations that can occur in the unspecified region of Figure 2. It is productive to first understand valid box configurations for $\mathcal{F}$ and then to precisely identify the $\mathcal{O}_{Y}$-module structure dictating which boxes generate which other ones. In other words, we first describe $\mathcal{F}$ as an element of $K_{\mathrm{T}}(Y)$ and then as an element of $\operatorname{Coh}_{\mathrm{T}}(Y)$. Because the description is more combinatorially involved than for DT or PT theory, we outline the main ingredients here.

- Definition 3 introduces rods and various terminology for them. They are the main new ingredient for BS theory, in comparison with DT and PT theory.

- Lemmas 7 and 8 characterise the rods that can appear in $\mathcal{F}$ via the restrictions $\mathcal{Q} \in \mathrm{T}$ and $\mathcal{F} \in \mathrm{T}^{\perp}$, respectively.

- Lemma 10 describes the way in which $\mathcal{F}\left(U_{a}\right)$ is built from boxes and rods in a single chart.

- Proposition 6 describes all possible $\mathcal{F}$, in the case of a single nontrivial external leg $\lambda_{a}=\square$. Such $\mathcal{F}$ are called local models and essentially consist of the single leg along with some rods.

- Proposition 12 characterises all possible $\mathcal{F}$ in general in terms of local models.

\subsection{4.}

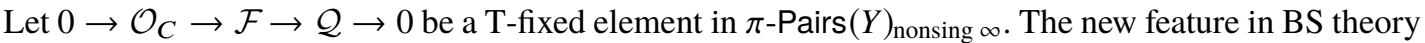
that is not present in DT or PT theories is the possibility of 1-dimensional components in $\mathcal{Q}$. Because $\mathcal{Q}$ is supported on $E$, we now introduce all of the necessary terminology for box configurations pertaining to 1-dimensional sheaves on $E$.

Definition 3. Let $E_{c} \cong \mathbb{P}^{1}$ be the components of the exceptional divisor $E$, and let $E_{a b}:=E_{a} \cup E_{a+1} \cup$ $\cdots \cup E_{b}$. Let

$$
\mathcal{R}:=\mathcal{O}_{E_{a b}}\left(d_{a}, d_{a+1}, \ldots, d_{b}\right)
$$

denote the line bundle on $E_{a b}$ such that $\left.\mathcal{R}\right|_{E_{c}}=\mathcal{O}_{\mathbb{P}^{1}}\left(d_{c}\right)$ for each $a \leq c \leq b$, with trivial gluing at nodes.

- A rod is a connected collection of boxes forming a line bundle of the form $\mathcal{R}$, with any linearisation.

- The length of the rod $\mathcal{R}$ is $b-a+1$, and its degrees are $\boldsymbol{d}(\mathcal{R}):=\left(\operatorname{deg}_{E_{c}} \mathcal{R}\right)_{a \leq c \leq b}$.

- In the chart $U_{a}$, we say the rod is pointing rightward; analogously, it points leftward in the chart $U_{b}$. Slightly abusing terminology, we say the leftmost/rightmost boxes in the rod generate the rod.

In Figure 3, the boxes coloured yellow form a $\operatorname{rod} \mathcal{R}$ of length 2 and degree $\boldsymbol{d}=(1,-1)$ generated by the box 四 $=z^{-1} \in U_{0}$. Alternatively, $\mathcal{R}$ is the equivariant line bundle $\mathcal{O}_{E}(1,-1)$ with linearisation

$$
\begin{aligned}
& \left.\mathcal{R}\right|_{p_{0}}=z^{-1} \\
& \left.\mathcal{R}\right|_{p_{1}}=y_{1}^{-1} z^{-1}=x y^{-2} z^{-1} \\
& \left.\mathcal{R}\right|_{p_{2}}=x_{2}^{-1} y_{2}^{-1} z^{-1}=x^{-1} y^{-1} z^{-1} .
\end{aligned}
$$




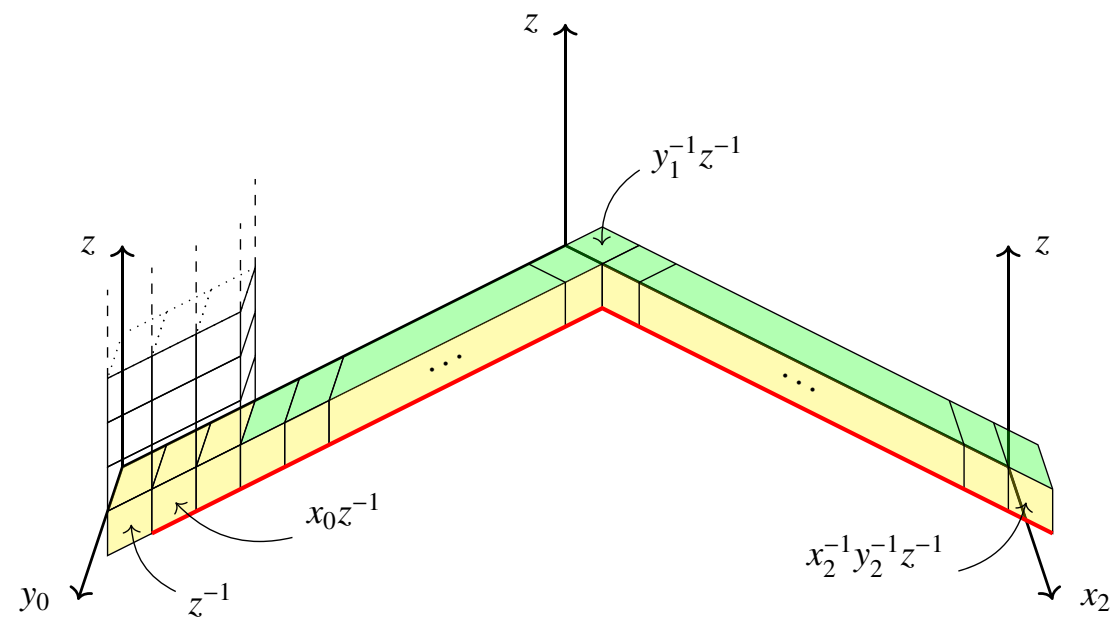

Figure 3. A T-fixed point in $\pi$-Pairs $(3), \emptyset, \emptyset\left(\mathcal{A}_{2}\right)_{\text {nonsing } \infty}$.

To relate weights with degrees, it is helpful to recall that on a $\mathbb{P}^{1}$ whose coordinate around 0 has weight $w$,

$$
\left.\mathcal{O}_{\mathbb{P}^{1}}(d)\right|_{\infty}=\left.w^{d} \mathcal{O}_{\mathbb{P}^{1}}(d)\right|_{0}
$$

For example, $\left.\mathcal{R}\right|_{p_{2}}=\left.x_{1}^{-1} \mathcal{R}\right|_{p_{1}}$ demonstrates that $\mathcal{R}$ has degree -1 on $E_{2}$.

Definition 4. A box in a rod $\mathcal{R}$ is exposed if it is $z$-torsion; that is, it generates only a finite stack of additional boxes in the $z$ direction. The exposed part of a rod forms possibly multiple disconnected rods.

○ A standard rightward rod is a rod with degrees $\boldsymbol{d}=(0,0, \ldots, 0,-1)$ and nonexposed generator on the left.

- A standard leftward rod is a rod with degrees $\boldsymbol{d}=(-1,0,0, \ldots, 0)$ and nonexposed generator on the right.

Standard rods are in some sense the minimal ones in $\mathrm{T}$; it is easiest to work only with standard rods for the combinatorial description of general $\mathcal{F}$.

In Figure 3, the exposed boxes in the rod $\mathcal{R}$ are shaded green on one face and form a rod $\mathcal{R}^{\prime}=$ $\mathcal{O}_{E}(-2,-1) \subset \mathcal{R}$ themselves (with appropriate linearisation). The rod $\mathcal{R}^{\prime}$ is generated by the box 四 $=x_{0}^{3} z^{-1}$. Note that $\mathcal{R}$ also has the subrod generated by $\left.⿴ 囗 ⿱ 一 一\right)=x_{0} z^{-1}$. This subrod is underlined in red in the figure and is a standard rightward rod of length 2.

\subsection{5.}

All 0 -dimensional sheaves on $Y$ are in $\mathrm{T}$, so sheaves in $\mathrm{T}^{\perp}$ are pure of dimension 1 . In particular, $\mathcal{F}$ and its subsheaf $\mathcal{O}_{C}$ are pure. Consequently, $C$ is Cohen-Macaulay, as in DT and PT theory. Thus, $\mathcal{O}_{C}$ contains all of the boxes in the infinite legs $L_{a}$, plus possibly some internal legs, and nothing more. For consistency, we view all internal legs in $\mathcal{O}_{C}$ as rods as well.

Whereas for stable pairs one can show that $C$ and the scheme-theoretic support $C_{\mathcal{F}}:=\operatorname{supp}(\mathcal{F})$ coincide [40, Lemma 1.6], this is very much not true for $\pi$-stable pairs, and in general,

$$
C^{\mathrm{red}} \neq C_{\mathcal{F}}^{\mathrm{red}}
$$

This is because $C_{\mathcal{F}}$ receives contributions from $\mathcal{Q}$, which may contain rods that are not present in $\mathcal{O}_{C}$. 
2.3.6.

Consider the case when all external legs are trivial except for $\lambda_{a}=\square$. Then we can give an explicit description of all possible $\mathcal{F}$. Importantly, the general case can be reduced to understanding this special case.

Definition 5. A local model for $\lambda_{a}=\square$ is an indecomposable sheaf $\mathcal{F} \in \operatorname{Coh}_{\mathrm{T}}(Y)$ that contains all boxes

$$
\left\{\mathbb{U}_{k}:=z^{k} \in U_{a}\right\}_{k \geq M}
$$

for some $M \in \mathbb{Z}$ (possibly with multiplicity), such that $\mathcal{F}$ consists only of $\left\{\mathbb{U}_{k}\right\}_{k \geq M}$ and finitely many rods generated by these boxes and

$\circ$ if $\mathbb{U}_{k}$ generates a rod, it must be a standard left- or rightward rod;

$\circ$ if $\mathbb{U}_{k}$ generates both a left- and rightward rod, then $\mathbb{U}_{k}$ has multiplicity 2 and the $\mathbb{U}_{k}$-weight space in $\mathcal{F}\left(U_{a}\right)$ is

$$
\left[z^{k}\right] \mathcal{F}\left(U_{a}\right)=\mathbb{C} v_{L} \oplus \mathbb{C} v_{R},
$$

where $v_{L}$ generates the leftward rod and $v_{R}$ generates the rightward rod;

$\circ$ if $\ell \in \mathbb{Z}$ is the largest integer such that $\mathbb{U}_{\ell}$ has multiplicity 2 , then the multiplication-by- $z$ map is

$$
\left[z^{\ell}\right] \mathcal{F}\left(U_{a}\right) \stackrel{(11)}{\longrightarrow}\left[z^{\ell+1}\right] \mathcal{F}\left(U_{a}\right) .
$$

Let level $(\mathcal{F}):=\ell$.

Figure 4 is the box diagram for a general local model $\mathcal{F}$; that $\mathcal{F}$ is a valid $\mathcal{O}_{Y}$-module forces the configuration of standard rods to be increasing in length as the degree in $z$ increases, as shown. For visual clarity, we have flattened the toric diagram and all boxes (cf. Figure 3).

Proposition 6 (Local model). Let $\mathcal{F} \in \pi$-Pairs ${ }_{\lambda}^{T}$ where $\lambda=\left(\cdots, \emptyset, \emptyset, \lambda_{a}=\square, \emptyset, \emptyset, \cdots\right)$. Then $\mathcal{F}$ is $a$ local model for $\square \in \lambda_{a}$ with level $(\mathcal{F})<0$.

For readers familiar with PT theory, the allowed box configurations for $\mathcal{F}$ are the same as for the PT 1-leg vertex for $\lambda=\square$, except now boxes are allowed to emit standard left-/rightward rods in such a way that $\mathcal{F}$ remains a valid $\mathcal{O}_{Y}$-module. When a box emits both left- and rightward rods, we will see that it necessarily gains a multiplicity.

\subsection{7.}

To begin the proof of Proposition 6, it is important to characterise the degrees/lengths of rods that can appear in $\mathcal{Q}$ and therefore in $\mathcal{F}$. Let $\mathcal{Q} \in \mathrm{T}$ be T-fixed, and let $\mathcal{R}$ be a rod in $\mathcal{Q}$. The condition that $\mathcal{Q} \in \mathrm{T}$ means that $H^{1}(E, \mathcal{R})=0$.

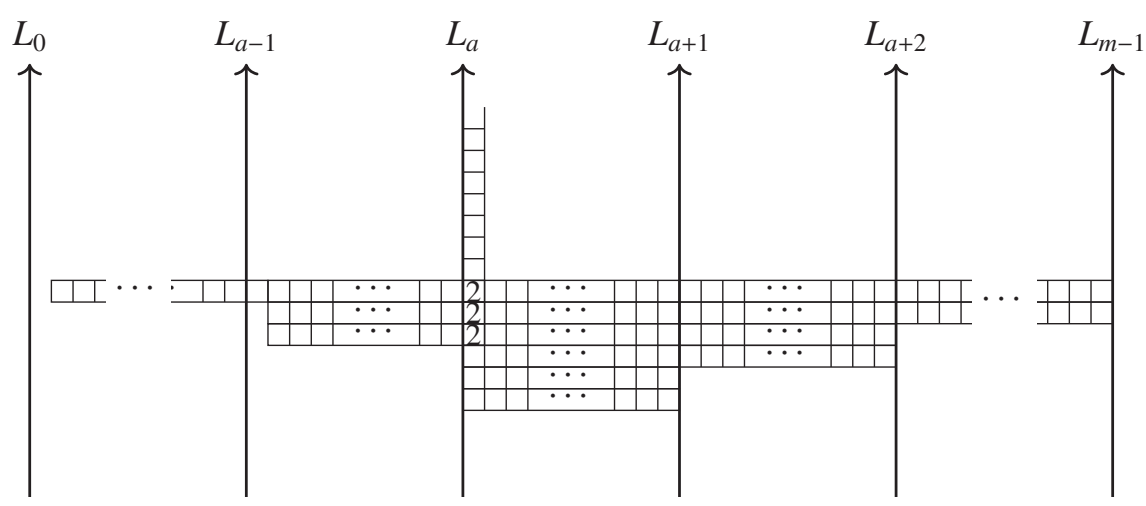

Figure 4. (Flattened) box diagram of a T-fixed pair with one nonzero leg $\lambda_{a}=\square$. 
Lemma 7. Let $\boldsymbol{d}(\mathcal{R})=\left(d_{a}, d_{a+1}, \ldots, d_{b}\right)$. Then $H^{1}(E, \mathcal{R})=0$ iff all $d_{c} \geq-1$ with equality for at most one $d_{c}$.

Proof. The exceptional divisor $E$ is a nodal chain of its components $E_{c} \cong \mathbb{P}^{1}$. Via normalisation, the $\operatorname{rod} \mathcal{R}$ fits into an exact sequence

$$
0 \rightarrow \mathcal{R} \rightarrow \bigoplus_{c=a}^{b} \mathcal{R}_{c} \rightarrow \mathcal{P} \rightarrow 0
$$

where $\mathcal{R}_{c} \cong \mathcal{O}\left(d_{c}\right)$ is supported only on $E_{c} \cong \mathbb{P}^{1}$ and $\mathcal{P}$ is supported only at the nodes $p_{a}$. The desired result follows from a standard argument using the associated long exact sequence.

\subsection{8.}

The condition that $\mathcal{F} \in \mathrm{T}^{\perp}$ also imposes a restriction on the lengths of rods. This is because no subsheaf $\mathcal{E}$ of an exposed rod can be an element of T. Otherwise, $\mathcal{F}$ would contain the subsheaf generated by $\mathcal{E}$, contradicting $\mathcal{F} \in \mathrm{T}^{\perp}$.

Lemma 8. Let $\mathcal{E}$ be an exposed rod of length $\ell$. Then it is a subsheaf of the maximal exposed rod $\mathcal{E}_{\text {max }, \ell}$ of length $\ell$, where

$\circ$ if $\ell>1$, then $\boldsymbol{d}\left(\mathcal{E}_{\max , \ell}\right)=(-1,0,0, \ldots, 0,0,-1)$;

$\circ$ if $\ell=1$, then $\boldsymbol{d}\left(\mathcal{E}_{\max , \ell}\right)=(-2)$.

Proof. Follows from the above discussion and Lemma 7 describing elements of $\mathrm{T}$. The maximal exposed rods $\mathcal{E}_{\max , \ell}$ are those that are maximal under inclusion; that is, such that increasing the degree on any component creates a subsheaf in $\mathrm{T}$.

When a sheaf $\mathcal{F}$ is pure of dimension 1, violating this lemma is the only way it can fail to be in $\mathrm{T}^{\perp}$. Note that some care must be taken when there are rods consisting of boxes with multiplicity. The following example shows that, in general, there cannot be two rods that start at the same box.

Example 9. Figure 5 contains an exposed rod of degree -1 , not just exposed rods of degree -2 . One way to see this exposed rod is to write down the vector space at each box along with the relevant module maps. In this case, the $\mathcal{O}_{Y}$-module structure is uniquely determined by the box configuration: one can always rescale the $\mathbb{C}^{2}$ in the rod so that the map $\mathbb{C}^{2} \rightarrow \mathbb{C}$ is as specified. Then the exposed rod of degree -1 is generated by the vector $\left(\begin{array}{c}1 \\ -1\end{array}\right)$ at each $\mathbb{C}^{2}$.

\subsection{9.}

The next step is to understand the manner in which $\mathcal{F}$ is built from boxes and rods. The following lemma shows that, aside from the presence of rods, $\mathcal{F}\left(U_{a}\right)$ is essentially a PT 1-leg vertex. Let $\mathcal{F}^{b} \subset \mathcal{F}$ be the subsheaf consisting only of boxes that are not part of rods, and similarly for $\mathcal{Q}^{\mathrm{b}}$. For short, let $\mathcal{F}_{a}$ denote $\mathcal{F}\left(U_{a}\right)$ and similarly for other sheaves.

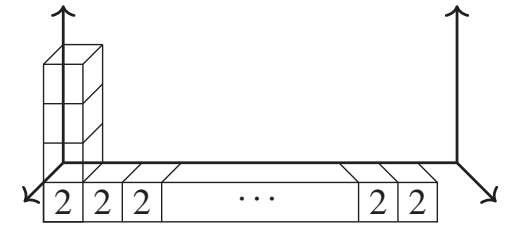

(a) Box diagram

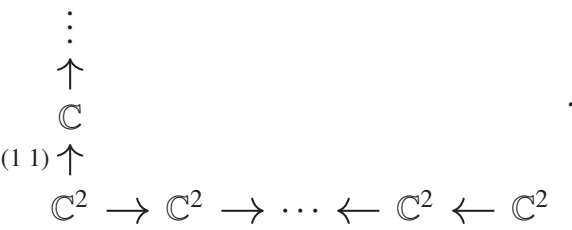

(b) $O_{Y}$-module structure

Figure 5. Non- $\pi$-stable: contains a degree -1 exposed rod. 
Lemma 10. In the chart $U_{a}$, the module $\mathcal{F}^{b}\left(U_{a}\right)$ is a T-invariant submodule of the localisation

$$
M_{a}:=\left(L_{a}\right)_{z}=\mathbb{C}\left[x_{a}, y_{a}, z, z^{-1}\right] / I_{\lambda_{a}}
$$

such that $z^{n} L_{a} \subset \mathcal{F}^{b}\left(U_{a}\right)$ for $n \gg 0$.

Proof. The key observation is that $\mathcal{F}_{a}^{\mathrm{b}}$ is $z$-torsion-free. This is because boxes in $\mathcal{F}_{a}^{\mathrm{b}}$ are necessarily torsion in the $x_{a}$ and $y_{a}$ directions, so if a box were also $z$-torsion then $\mathcal{F}$ would fail to be pure of dimension 1.

The only way for a box 四 $\in \mathcal{F}_{a}$ to be $z$-torsion-free is to have $z^{n}$. 四 $\in L_{a}$ for $n \gg 0$. Hence, the composition

$$
\mathcal{F}_{a}^{\mathrm{b}} \hookrightarrow \mathcal{F}_{a} \rightarrow \operatorname{Hom}\left(\operatorname{Hom}\left(\mathcal{F}_{a}, L_{a}\right), L_{a}\right)
$$

is generically an isomorphism and therefore an inclusion by the purity of $\mathcal{F}_{a}^{b}$. To compute $\operatorname{Hom}\left(\operatorname{Hom}\left(\mathcal{F}_{a}, L_{a}\right), L_{a}\right)$, apply $\operatorname{Hom}\left(-, L_{a}\right)$ to $0 \rightarrow\left(\mathcal{O}_{C}\right)_{a} \rightarrow \mathcal{F}_{a} \rightarrow \mathcal{Q}_{a} \rightarrow 0$. Because everything in $\mathcal{Q}_{a}$ is $z$-torsion, this yields an inclusion

$$
0 \rightarrow \operatorname{Hom}\left(\mathcal{F}_{a}, L_{a}\right) \rightarrow \operatorname{Hom}\left(\left(\mathcal{O}_{C}\right)_{a}, L_{a}\right)=L_{a}
$$

So $\operatorname{Hom}\left(\mathcal{F}_{a}, L_{a}\right)$ is an ideal $I_{Z} \subset L_{a}$. The subscheme $Z$ it cuts out must be 0 -dimensional because $\operatorname{supp} \mathcal{Q}_{a}^{\mathrm{b}}$ is. Thus, $\operatorname{Hom}\left(I_{Z}, L_{a}\right)$ is a submodule of $M_{a}$ satisfying the specified criterion.

Remark 11. Let $\mathcal{F}^{z}$ be the quotient of $\mathcal{F}$ consisting of boxes that are $z$-torsion-free. Then there is a short exact sequence

$$
0 \rightarrow \mathcal{F}^{\mathrm{b}} \rightarrow \mathcal{F}^{z} \rightarrow \mathcal{F}^{z, \mathrm{r}} \rightarrow 0
$$

where $\mathcal{F}^{z, \mathrm{r}}$ consists of $z$-torsion-free boxes that are part of rods. Every rod must contain some boxes that are $z$-torsion-free; otherwise, the subsheaf of $\mathcal{F}$ generated by the pre-image of the rod is also an element in $\mathrm{T}$, contradicting $\mathcal{F} \in \mathrm{T}^{\perp}$.

As with boxes in $\mathcal{F}^{b}$, boxes 四 $\in \mathcal{F}_{a}^{z, \mathrm{r}}$ must have $z^{n}$.⿴囗十 $\in L_{a}$ for $n \gg 0$. However, $\mathcal{F}_{a}^{z}$ is not necessarily a submodule of $M_{a}$; the proof of Lemma 10 fails because $\mathcal{F}^{z}$ is not a subsheaf of $\mathcal{F}$. This therefore allows for boxes with multiplicity to occur in $\mathcal{F}^{z, r}$.

\subsubsection{0.}

We can now give the proof of Proposition 6, which describes all possible local models $\mathcal{F}$.

Proof (of Proposition 6). By Lemmas 7 and 8, the only rods that can appear in a local model are standard rods. For example, the simplest case of a rightward rod of length 1 must have degree $\geq-1$ and its exposed part must have degree $\leq-2$. Therefore, it must be exactly of degree -1 ; that is, a standard rod.

By the discussion of Subsection 2.3.9, these standard rods must be generated by boxes $⿴ 囗 十$ that are $z$-torsion-free, which therefore eventually generate $L_{a}$. Because $\mathcal{F}$ is an $\mathcal{O}_{Y}$-module, once a standard rod appears, all rods that are stacked on top of it in the $z$ direction must have nondecreasing length. Finally, note that a single box cannot generate both leftward and rightward rods; such a rod would violate Lemma 7 and is not in T. Hence, if a leftward and a rightward standard rod start at the same box, that box has multiplicity 2 . Taking all of these constraints into account, $\mathcal{F}$ must be as described. The restriction on level $(\mathcal{F})$ arises because there must be an inclusion $\mathcal{O}_{C} \hookrightarrow \mathcal{F}$, where here $C=\left\{p_{a}\right\} \times \mathbb{C}_{z}$.

\subsubsection{1.}

The description in Proposition 6 of the local model is not specific to the $\square_{0}:=x_{a}^{0} y_{a}^{0}$ column in $L_{a}$. In general, a local model for $\square=x_{a}^{i} y_{a}^{j} \in \lambda_{a}$ is given by multiplying a local model for $\square_{0}$ by $\square$. 
Proposition 12. Let $\left[\mathcal{O}_{Y} \rightarrow \mathcal{F}\right]$ be a $T$-fixed $\pi$-stable pair with legs $\lambda$. Then there is a (not necessarily unique) filtration of length $n=|\lambda|$

$$
\emptyset=\mathcal{F}_{n} \subset \mathcal{F}_{n-1} \subset \cdots \subset \mathcal{F}_{0}=\mathcal{F}
$$

whose associated graded pieces are local models for each $\square \in \lambda_{a}$ (for $\left.0 \leq a<m\right)$.

Proof. Pick the smallest $a$ such that $\lambda_{a}$ is nonempty. Pick $\square \in \lambda_{a}$ not supporting any other squares in $\lambda_{a}$. Let $\mathcal{G}$ be the largest local model at $\square$ that is still a subsheaf of $\mathcal{F}$. By largest we mean with standard leftward and rightward rods of maximal possible length generated at every box $z^{k} \cdot \square$. The claim is that the resulting quotient $\mathcal{F}^{\prime}:=\mathcal{F} / \mathcal{G}$ is still $\pi$-stable. Then $\mathcal{F}^{\prime}$ contains strictly fewer columns in its infinite legs, and induction with Proposition 6 as the base case finishes the proof.

Let $0 \rightarrow \mathcal{O}_{C^{\prime}} \rightarrow \mathcal{F}^{\prime} \rightarrow \mathcal{Q}^{\prime} \rightarrow 0$ be the resulting pair. There are two ways $\mathcal{F}^{\prime}$ can fail to be $\pi$-stable: $\mathcal{Q}^{\prime} \notin \mathrm{T}$ or $\mathcal{F}^{\prime} \notin \mathrm{T}^{\perp}$. Clearly, $\mathcal{Q}^{\prime} \in \mathrm{T}$ iff $\mathcal{Q} \in \mathrm{T}$, because all standard rods satisfy Lemma 7 . So suppose $\mathcal{F}^{\prime} \notin \mathrm{T}^{\perp}$. Then $\operatorname{Hom}\left(\mathcal{T}, \mathcal{F}^{\prime}\right) \neq 0$ for some $\mathcal{T} \in \mathrm{T}$. If $\operatorname{dim} \operatorname{supp} \mathcal{T}=0$, then the image of $\mathcal{T}$ in $\mathcal{F}^{\prime}$ must consist of boxes extending some standard rod that was removed as part of $\mathcal{G}$. Then there is an exposed rod in $\mathcal{F}$ violating Lemma 8, contradicting $\mathcal{F} \in \mathrm{T}^{\perp}$.

The only remaining possibility is that $\operatorname{dim} \operatorname{supp} \mathcal{T}=1$. Namely, removing $\mathcal{G}$ created an exposed rod $\mathcal{R}^{\prime}$ in $\mathcal{F}^{\prime}$ whose pre-image in $\mathcal{F}$ is not an exposed rod. Let $\left.⿴ 囗 ⿱ 一 一\right)$ denote the box in $\mathcal{R}^{\prime}$ lying in the column corresponding to $\square$. By Lemma 8, making 四 into a $z$-torsion-free box turns $\mathcal{R}^{\prime}$ into a standard $\operatorname{rod} \mathcal{R}$ in $\mathcal{F}$, which should have been removed as part of $\mathcal{G}$. The only way for it to still be part of $\mathcal{F}^{\prime}$ is if it overlaps with a standard rod already in $\mathcal{G}$, in the form of boxes with multiplicity in $\mathcal{R}$. As in Example 9 , these multiplicity $>1$ boxes therefore contain an exposed rod violating Lemma 8, again contradicting $\mathcal{F} \in \mathrm{T}^{\perp}$.

\subsubsection{2.}

The converse to Proposition 12 is also true and important for us when we want to build $\pi$-stable pairs out of specific local models.

Proposition 13. Suppose $\mathcal{F} \in \operatorname{Coh}(Y)$ admits an inclusion $\mathcal{O}_{C} \hookrightarrow \mathcal{F}$ where $C$ has external legs $\lambda$ and that it also admits a filtration

$$
\emptyset=\mathcal{F}_{n} \subset \mathcal{F}_{n-1} \subset \cdots \subset \mathcal{F}_{0}=\mathcal{F}
$$

of length $n=|\lambda|$ whose associated graded pieces are local models for each $\square \in \lambda_{a}$ (for $\left.0 \leq a<m\right)$. Then the induced

$$
s: \mathcal{O}_{Y} \rightarrow \mathcal{O}_{C} \hookrightarrow \mathcal{F}
$$

is a $\pi$-stable pair.

Proof. We engineered the notion of a local model to satisfy the constraints of Lemma 7 and Lemma 8 for $\mathcal{Q}$ and $\mathcal{F}$ to be in $\mathrm{T}$ and $\mathrm{T}^{\perp}$, respectively.

\subsection{T-fixed loci}

\subsection{1.}

In general, the T-fixed loci in $\pi$-Pairs can have arbitrarily large dimension. Because the virtual tangent space (7) for $\pi$-Pairs depends only on the box configuration and there is no ambiguity in forming a local model from a given box configuration, these fixed loci must arise from the ambiguity in reconstructing the $\mathcal{O}_{Y}$-module $\mathcal{F}$ from the local models. More precisely, note that for fixed $a$, the local models for each $\square \in \lambda_{a}$ form a $\mathcal{O}_{Y}$-module $\mathcal{F}_{a}$ uniquely (cf. the proof of Lemma 43), essentially because the $\mathcal{O}_{Y}$-module maps between boxes in different local models are induced from those of the leg $L_{a}$. By Proposition 12 , the ambiguity in reconstructing $\mathcal{F}$ is therefore solely in how to attach different $\mathcal{F}_{a}$ to 


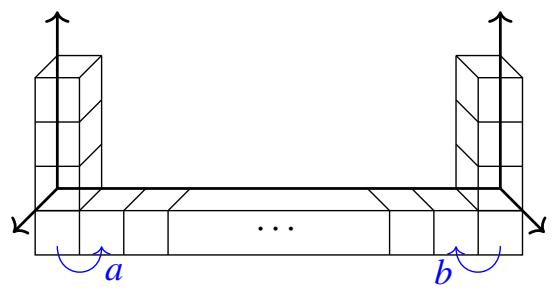

Figure 6. Box configuration for a positive-dimensional fixed locus.

each other. In other words, given fixed $\mathcal{F}_{a}$ for $0 \leq a<m$, the space of all possible $\mathcal{F}$ is controlled by the groups $\operatorname{Ext}^{1}\left(\mathcal{F}_{a}, \mathcal{F}_{b}\right)$ for $0 \leq a, b<m$.

\subsection{2.}

In particular, all nontrivial extensions between $\mathcal{F}_{a}$ and $\mathcal{F}_{b}$ with $a<b$ arise when there are two boxes 四 $\in \mathcal{F}_{a}$ and 四 $\in \mathcal{F}_{b}$ with the same T-weight and either 四 $\in \mathcal{F}_{a}$ generates a standard rightward rod or 四 $\in \mathcal{F}_{b}$ generates a standard leftward rod. The simplest example is as follows.

Example 14. Consider the vertex for $(\square, \square) \in \mathcal{A}_{1}^{\mathrm{T}}$. One T-fixed component for this vertex has the following box configuration. Here the $a$ and $b$ arrows represent $\mathcal{O}_{Y}$-module maps. Note that every point $[a: b] \in \mathbb{P}^{1}$ yields a distinct $\mathcal{O}_{Y}$-module $\mathcal{F}$. The points $0 \in \mathbb{P}^{1}$ and $\infty \in \mathbb{P}^{1}$ correspond to the direct sum of two local models: one with a single box and another with a standard leftward/rightward rod. The point $[1: 1]$ corresponds to $z^{-1} \mathcal{O}_{C}$, where $C$ is the $C M$ curve with the two specified external legs and one internal leg.

\subsection{3.}

The existence of positive-dimensional fixed loci should not be surprising, because already in PT theory fixed loci can be arbitrary products of $\mathbb{P}^{1}$ 's [39, Theorem 1]. Note, however, that it is heavily dependent on the underlying 3-fold geometry being of the form $\operatorname{tot}\left(\mathcal{O}_{\mathbb{P}^{1}} \oplus \mathcal{O}_{\mathbb{P}^{1}}(d)\right)$; that is, having a trivial zdirection. When this is not the case, such as for the $\operatorname{conifold} \operatorname{tot}(\mathcal{O}(-1) \oplus \mathcal{O}(-1))$, the fixed locus should consist of only isolated fixed points.

That the fixed loci are always proper will follow from the properness of the corresponding quasimap fixed loci and the isomorphism of fixed loci constructed in the BS/quasimaps correspondence. It seems difficult to prove properness directly in $\pi$-Pairs.

\section{Two Hilbert schemes}

\subsection{As Nakajima quiver varieties}

\subsection{1.}

Let $Q$ be a quiver with vertex set $I$. The associated Nakajima quiver variety $\mathcal{M}_{\boldsymbol{\theta}}$ depends on a GIT stability parameter $\boldsymbol{\theta} \in \mathbb{R}^{I}$. We briefly review the construction.

Definition 15 ([31, Section 2], [15, Section 5]). For two $I$-graded vector spaces $V=\bigoplus_{i \in I} V_{i}$ and $W=\bigoplus_{i \in I} W_{i}$, let

$$
\operatorname{Rep}_{Q}^{\mathrm{framed}}:=\bigoplus_{\text {edge } i \rightarrow j} \operatorname{Hom}\left(V_{i}, V_{j}\right) \oplus \bigoplus_{i \in I} \operatorname{Hom}\left(W_{i}, V_{i}\right)
$$

be the space of framed representations of the quiver $Q$, where $W$ is the framing. Then $T^{*} \operatorname{Rep}{ }_{Q}^{\text {framed }}$ has a standard symplectic form and the standard action by $G_{V}:=\prod_{i \in I} \mathrm{GL}\left(V_{i}\right)$ is Hamiltonian with moment 
map, which we denote $\mu$. The GIT quotient

$$
\mathcal{M}_{\theta}:=\mu^{-1}(0) / /{ }_{\theta} G_{V}
$$

is called the algebraic symplectic reduction. The resulting Nakajima quiver variety has components $\mathcal{M}_{\boldsymbol{\theta}}(\boldsymbol{v}, \boldsymbol{w})$ indexed by the dimension vectors $\boldsymbol{v}$ and $\boldsymbol{w}$ of $V$ and $W$.

When $\boldsymbol{\theta}$ is generic, $\mathcal{M}_{\boldsymbol{\theta}}$ is smooth and is a fine moduli space of quiver representations. It therefore has a universal family

$$
V=\bigoplus_{i \in I} V_{i}
$$

(The same goes for $W=\bigoplus_{i \in I} W_{i}$, which is a trivial bundle and less interesting.)

\subsection{2.}

By the general theory of GIT quotients, $\mathcal{M}_{\boldsymbol{\theta}}(\boldsymbol{v}, \boldsymbol{w})$ is actually independent of $\boldsymbol{\theta}$ within chambers in $\mathbb{R}^{I}$ cut out by a certain hyperplane arrangement. For Nakajima quiver varieties, these chambers are exactly the Weyl chambers of the (generalised) Kac-Moody algebra associated to $Q$.

The varieties $\mathcal{M}_{\boldsymbol{\theta}}$ and $\mathcal{M}_{\boldsymbol{\theta}^{\prime}}$ for $\boldsymbol{\theta}$ and $\boldsymbol{\theta}^{\prime}$ in different stability chambers are related as follows. Let $(B, i) \in \operatorname{Rep}_{Q}^{\text {framed }}$ and $(\bar{B}, j)$ be in the cotangent fibre. Points in $\mathcal{M}_{\theta}$ are represented by tuples $(B, \bar{B}, i, j)$ satisfying the moment map equation $\mu=0$ and the stability condition imposed by $\boldsymbol{\theta}$. There is an $S^{1}$ action given by

$$
t \cdot(B, \bar{B}, i, j):=\left(t B, t^{-1} \bar{B}, i, j\right)
$$

that respects the equation $\mu=0$ but may change stability chambers.

Theorem 16 ([30, 4.1.3]). For generic $\boldsymbol{\theta}, \boldsymbol{\theta}^{\prime}$, the varieties $\mathcal{M}_{\boldsymbol{\theta}}$ and $\mathcal{M}_{\boldsymbol{\theta}^{\prime}}$ are $S^{1}$-equivariantly diffeomorphic.

Remark 17. For algebraic symplectic reductions, it is straightforward to verify that both $\boldsymbol{\theta}$ and $-\boldsymbol{\theta}$ yield isomorphic spaces. Due to this, our stability chambers/criteria may differ from convention by a sign.

\subsection{3.}

The quiver $Q$ we are interested in arises from the McKay correspondence, which associates to every surface singularity of type ADE an affine Dynkin diagram of the corresponding type.

Definition 18. If $\Gamma \subset \operatorname{SL}(2, \mathbb{C})$ defines the singularity, let $R_{0}=\mathbb{C}, R_{1}, \ldots, R_{m-1}$ denote the irreducible representations of $\Gamma$ and $\mathbb{C}^{2}$ be the natural representation of $\Gamma$. Decompose

$$
R_{i} \otimes \mathbb{C}^{2} \cong \bigoplus R_{j}^{\oplus a_{i j}}
$$

Then the matrix $A:=\left(a_{i j}\right)$ is the adjacency matrix for the Dynkin diagram.

For $\mathcal{A}_{m-1}$, the resulting quiver is the affine (or cyclic) type A quiver in Figure 7. After framing and doubling, we view $T^{*} \operatorname{Rep}_{Q}^{\text {framed }}$ as the moduli of representations of the quiver shown in Figure 7 . The dimension vectors

$$
\boldsymbol{v}^{0}:=\left(\operatorname{dim} R_{0}, \operatorname{dim} R_{1}, \ldots, \operatorname{dim} R_{m-1}\right), \quad \boldsymbol{w}^{0}:=(1,0,0, \ldots, 0)
$$

will be especially relevant. For $\mathcal{A}_{m-1}$, clearly $\operatorname{dim} R_{i}=1$ for all $i$. In Lie-theoretic language, $v^{0}$ is the affine root of the associated affine Lie algebra. 


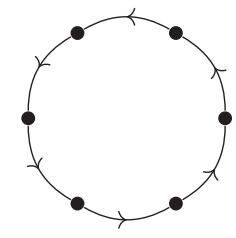

(a) Affine type A quiver

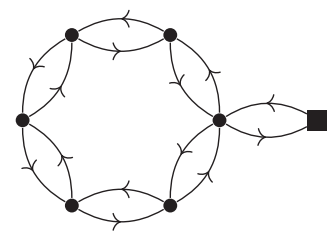

(b) Doubled framed affine type A quiver

Figure 7. Quivers associated to the $A_{5}$ surface singularity.

3.1.4.

For affine ADE quivers, there are two stability chambers we are interested in. The first is

$$
C_{+}:=\left\{\boldsymbol{\theta} \in \mathbb{R}^{m} \mid \theta_{i}>0 \text { for all } 0 \leq i<m\right\} .
$$

The following theorem rephrases a well-known generalisation of the description of $\operatorname{Hilb}\left(\mathbb{C}^{2}\right)$ as a Nakajima quiver variety.

Theorem 19 ([32, Theorem 4.4]). For any integer $n \geq 0$ and any $\boldsymbol{\theta} \in C_{+}$, there is an isomorphism

$$
\mathcal{M}_{\boldsymbol{\theta}}\left(n \boldsymbol{v}^{0}, \boldsymbol{w}^{0}\right) \cong \operatorname{Hilb}^{n}\left(\left[\mathbb{C}^{2} / \Gamma\right]\right) .
$$

Here, the Hilbert scheme of $n$ points on the stack $\left[\mathbb{C}^{2} / \Gamma\right]$ is equivalently the Hilbert scheme of collections of $n m$ points on $\mathbb{C}^{2}$ that are $\Gamma$-fixed. For this reason, it is sometimes called the $\Gamma$-equivariant Hilbert scheme. In this language, a $\Gamma$-invariant ideal $I \subset \mathbb{C}[x, y]$ cutting out the $n m$ points corresponds to the quiver data

$$
\begin{aligned}
V_{k} & =\operatorname{Hom}_{\Gamma}\left(R_{k}, \mathbb{C}[x, y] / I\right) \\
W_{0} & =\mathbb{C} .
\end{aligned}
$$

The quiver maps are given by $i(1):=[1] \in \mathbb{C}[x, y] / I$ and $j:=0$, and the $B, \bar{B}: V_{i} \rightarrow V_{j}$ are multiplication by $x$ or $y$, as per the McKay correspondence. For the affine type A quiver defining $\mathcal{A}_{m-1}$, all counterclockwise arrows are multiplication by $x$ and all clockwise arrows are multiplication by $y$.

\subsection{5.}

The second stability chamber we want is given in terms of the level-0 hyperplane

$$
D_{\delta}:=\left\{\boldsymbol{\theta} \in \mathbb{R}^{m} \mid \boldsymbol{v}^{0} \cdot \boldsymbol{\theta}=0\right\} .
$$

On $D_{\delta}$, there is a chamber structure defined by hyperplanes $D_{\alpha}:=\{\alpha \cdot \boldsymbol{\theta}=0\}$ for finite roots $\alpha$; in particular,

$$
C:=\left\{\boldsymbol{\theta} \in D_{\delta} \mid \theta_{i}>0 \text { for all } 1 \leq i<m\right\}
$$

is a chamber. Let $C_{-}(m)$ be the unique chamber in $\mathbb{R}^{m}$ lying on the positive side $\left\{\boldsymbol{v}^{0} \cdot \boldsymbol{\theta}>0\right\}$ of $D_{\delta}$ with $C$ as its face. For example, in type A,

$$
(-m+1+\epsilon, 1,1, \ldots, 1) \in C_{-}(m)
$$

for sufficiently small $\epsilon>0$.

Theorem 20 ([33, Theorem 4.2], [24, Theorem 4.9]). For any integer $n \geq 0$ and $\boldsymbol{\theta} \in C_{-}(m)$, there is an isomorphism

$$
\mathcal{M}_{\boldsymbol{\theta}}\left(n \boldsymbol{v}^{0}, \boldsymbol{w}^{0}\right) \cong \operatorname{Hilb}^{n}(S)
$$

where $S \rightarrow \mathbb{C}^{2} / \Gamma$ is the minimal resolution. 
Let $Z \subset \mathcal{A}_{m-1}$ be a length- $n$ subscheme. Then the corresponding quiver data is

$$
\begin{aligned}
V_{k} & =H^{0}\left(\mathcal{A}_{m-1}, V_{k} \otimes \mathcal{O}_{Z}\right) \\
W_{0} & =H^{0}\left(\mathcal{A}_{m-1}, \mathcal{O}_{\mathcal{A}_{m-1}}\right)=\mathbb{C},
\end{aligned}
$$

where $V=\bigoplus V_{k}$ is the universal family for $\operatorname{Hilb}^{1}\left(\mathcal{A}_{m-1}\right)=\mathcal{A}_{m-1}$. The quiver maps between the $V_{k}$ are induced from the quiver maps of the universal family. For the framing, $j=0: V_{0} \rightarrow W_{0}$ always whereas $i: W_{0} \rightarrow V_{0}$ is more subtle. If $\left(V_{0}\right)_{0} \subset V_{0}$ denotes the subspace of trivial $\mathrm{T}^{\prime}$-weight, then for $\operatorname{Hilb}\left(\left[\mathbb{C}^{2} / \Gamma\right]\right)$ we have $\operatorname{dim}\left(V_{0}\right)_{0}=1$ with generator $i(1)$. But for $\operatorname{Hilb}\left(\mathcal{A}_{m-1}\right)$, it turns out that $\operatorname{dim}\left(V_{0}\right)_{0}>1$ in general (see Subsection 3.2.5), in which case the framing is given by the diagonal map $i(1)=(1,1, \ldots, 1) \in\left(V_{0}\right)_{0}$.

\subsection{T-equivariant geometry}

\subsection{1.}

Both $\operatorname{Hilb}\left(\left[\mathbb{C}^{2} / \Gamma\right]\right)$ and $\operatorname{Hilb}\left(\mathcal{A}_{m-1}\right)$ inherit the standard action of the torus $\mathrm{T}^{\prime}$ on $\mathbb{C}^{2}$, from Subsection 2.1.2. K-theoretic computations on these spaces require understanding $T^{\prime}$-fixed points and their tangent spaces. In particular, for quasimap theory, it is productive to work with both Hilbert schemes as moduli of quiver representations, and therefore it is necessary to determine the quiver data $V=\bigoplus V_{k}$ at each fixed point.

3.2.2.

The $\Gamma$-equivariant Hilbert scheme of $n$ points is a subscheme

$$
\operatorname{Hilb}^{n}\left(\left[\mathbb{C}^{2} / \Gamma\right]\right) \subset \operatorname{Hilb}^{n m}\left(\mathbb{C}^{2}\right) .
$$

Hence, $\mathrm{T}^{\prime}$-fixed points in $\operatorname{Hilb}^{n}\left(\left[\mathbb{C}^{2} / \Gamma\right]\right)$ can be identified with certain Young diagrams of size $n m$.

Definition 21. The colour or content of a square $\square=x^{i} y^{j} \in \lambda$ is

$$
c(\square):=i(\square)-j(\square) \in \mathbb{Z} / m=\Gamma
$$

and records its $\Gamma$-weight. A uniformly coloured Young diagram $\lambda$ has the same number of squares of each colour.

Let $\lambda \in \operatorname{Hilb}^{n}\left(\left[\mathbb{C}^{2} / \Gamma\right]\right)^{\mathrm{T}^{\prime}}$ be a fixed point. The $\Gamma$-invariance of $\lambda$ implies that it is uniformly coloured. From (13), $V_{k}$ is therefore the character of all colour- $k$ boxes in $\lambda$. The quiver data for $\lambda$ will be denoted

$$
V(\lambda)=\bigoplus_{k} V_{k}(\lambda)
$$

\subsection{3.}

Similarly, a $\mathrm{T}^{\prime}$-fixed point $Z \in \operatorname{Hilb}^{n}\left(\mathcal{A}_{m-1}\right)$ is a collection of $m$ Young diagrams $\lambda_{0}, \ldots, \lambda_{m-1}$, one for each $\mathrm{T}^{\prime}$-fixed point $p_{i} \in \mathcal{A}_{m-1}$, so that

$$
\left.\mathcal{O}_{Z}\right|_{U_{a}}=\mathbb{C}\left[x_{a}, y_{a}\right] / I\left(\lambda_{a}\right)
$$

The ideal $I\left(\lambda_{a}\right)$ has generators $\left\{x_{a}^{i(\square)} y_{a}^{j(\square)}\right\}_{\square \in \lambda_{a}}$. Because $Z$ has length $n$ we require $\sum_{a=0}^{m-1}\left|\lambda_{a}\right|=n$, but otherwise the $\lambda_{a}$ can be arbitrary Young diagrams. From now on, we will abuse notation and write

$$
Z=\lambda:=\left(\lambda_{0}, \ldots, \lambda_{m-1}\right)
$$


to denote the fixed point. The quiver data for $\lambda$ will be denoted

$$
V(\lambda)=\bigoplus_{a} V_{a}(\lambda)
$$

To describe $V_{a}(\lambda)$ combinatorially, using (15), it remains to determine the universal family $V$ on $\mathcal{A}_{m-1}$.

\subsection{4.}

Because $\mathcal{A}_{m-1}$ is toric, an easy way to determine (equivariantly) the universal line bundles $V_{k}$ on $\mathcal{A}_{m-1}$ is to identify their weights at the fixed points $p_{a}$. This can be done from first principles by explicitly determining the corresponding $\mathrm{T}^{\prime}$-fixed quiver representations.

Proposition 22. For $0 \leq a, k<m$,

$$
\left.V_{k}\right|_{p_{a}}= \begin{cases}y^{m-k} & a<k \\ x^{k} & a \geq k\end{cases}
$$

nonequivariantly, which means $V_{0}=\mathcal{O}_{\mathcal{A}_{m-1}}$ and $V_{k}=\mathcal{O}_{E_{k}}$ (1) for $1 \leq k<m$. This is in agreement with [23, Lemma 2.1], where it is shown that $V_{k}=\mathcal{O}_{E_{k}}(1) \oplus \mathcal{O}_{E_{k}}^{\text {dim } R_{k}-1}$ for $k \neq 0$ in the general ADE case.

Proof. The dimension vector is $\boldsymbol{v}^{0}=(1,1, \ldots, 1)$, so all vector spaces $V_{i}$ are 1-dimensional. In this setting, the key observation is that nontrivial cycles in the quiver - namely, any composition of arrows that starts and ends at the same vertex $v$ - must be zero. Such a nonzero cycle would have a nontrivial $\mathrm{T}^{\prime}$-weight that cannot be cancelled by the $G_{V}$ freedom at $v$ and therefore cannot be $\mathrm{T}^{\prime}$-fixed. Hence, only trees can be $\mathrm{T}^{\prime}$-fixed, and it suffices to figure out which maps $B_{i j}$ or $\bar{B}_{i j}$ are nonzero. (The $G_{V}$ freedom can be used to scale all maps in nonzero trees to 1.)

GIT stability for framed quiver representations has a reformulation in terms of subobject stability, by work of King and Crawley-Bovey [15, Section 3.2]. In our setting, we need to extend the stability condition by $\theta_{\infty}:=-\boldsymbol{\theta} \cdot \operatorname{dim} V$ to include the extra framing vertex $W_{0}$. Then the reformulated stability criterion is that

$$
\left(\boldsymbol{\theta}, \theta_{\infty}\right) \cdot\left(\operatorname{dim} V^{\prime}, \operatorname{dim} W_{0}^{\prime}\right)>0
$$

for nontrivial subrepresentations $\left(V^{\prime}, W_{0}^{\prime}\right)$ (see the proof of [36, Lemma 7.2.10]). For us, using (14), this extended stability condition is

$$
\left(\boldsymbol{\theta}, \theta_{\infty}\right)=(-m+1+\epsilon, 1,1, \ldots, 1,-\epsilon),
$$

and $\operatorname{dim} V_{0}^{\prime} \in\{0,1\}$. It follows that $V_{0}$ must generate all other $V_{k}$. So the only $\mathrm{T}^{\prime}$-fixed quiver representations are those in Figure 8, with characters

$$
\mathrm{hk}_{a}:=y^{m-a}+y^{m-a-1}+\cdots+y+1+x+\cdots+x^{a}
$$
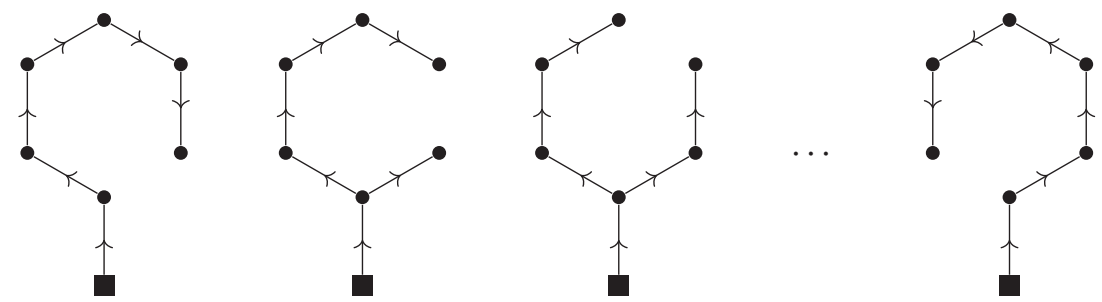

Figure 8. $T^{\prime}$-fixed quiver representations for $\mathcal{A}_{5}$ (only nonzero maps shown). 
for $0 \leq a<m$. Each of these characters is the restriction of the universal family $V$ to a fixed point $p_{a}$; the line bundle $V_{k}$ is the summand with $\mathbb{Z} / m$-weight $k$.

\subsection{5.}

Plugging (16) and (17) into (15) yields the following description for $V=\bigoplus_{k} V_{k}$. Given a fixed point $\lambda=\left(\lambda_{0}, \ldots, \lambda_{m-1}\right) \in \operatorname{Hilb}\left(\mathcal{A}_{m-1}, n\right)$,

$$
V(\lambda)=\sum_{a=0}^{m-1} V^{(a)}(\lambda), \quad V^{(a)}(\lambda):=\mathrm{hk}_{a} \sum_{\square \in \lambda_{a}} x_{a}^{i(\square)} y_{a}^{j(\square)} .
$$

In terms of diagrams, this means to take each $\lambda_{a}$ and draw it using $\left(x_{a}, y_{a}\right)$ coordinates but with each box replaced with the $m$-hook $\mathrm{hk}_{a}$ from (19). Then $V$ is the sum of all of the resulting diagrams. Figure 9 illustrates an example. Note that $V$ may have multiple boxes with the same $\mathrm{T}^{\prime}$-weight, which is not a phenomenon that occurs for $\operatorname{Hilb}\left(\left[\mathbb{C}^{2} / \Gamma\right]\right)$. We indicate this on diagrams by labelling boxes with their multiplicities whenever the multiplicity is greater than 1 .

As a quiver representation, $V(\lambda)$ is clearly also a sum of the quiver representations $V^{(a)}(\lambda)$. Each of these quiver representations has a nonzero multiplication by $x$ (respectively $y$ ) map whenever a box has a neighbouring box to its right (respectively above it). In other words, each box in $V^{(a)}(\lambda)$ generates all boxes above and to the right of it.

Example 23. The quiver representation corresponding to Figure 9 is

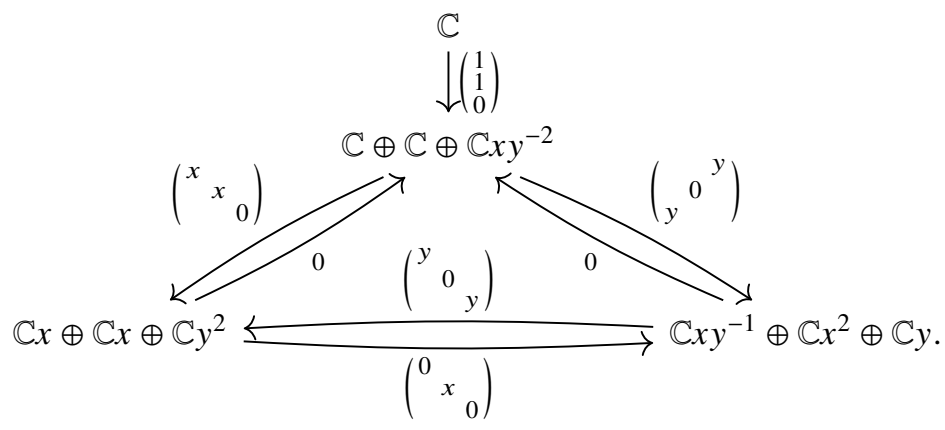

\subsection{6.}

In fact, $\mathrm{T}^{\prime}$-fixed points for $\operatorname{Hilb}\left(\left[\mathbb{C}^{2} / \Gamma\right]\right)$ and $\operatorname{Hilb}\left(\mathcal{A}_{m-1}\right)$ have a strong relationship. Theorem 16 implies that there is a bijection between $\mathrm{T}^{\prime}$-fixed points of these two spaces that is $\mathrm{A}^{\prime}$-equivariant; that is, the $\mathrm{A}^{\prime}$-characters of the corresponding quiver data must be equal. This bijection was described nonequivariantly in [24, Theorem 5.3] and $\mathrm{A}^{\prime}$-equivariantly in [30, Theorem 4.5], but now we can also see how the fully equivariant $\mathrm{T}^{\prime}$-characters of fixed points compare.

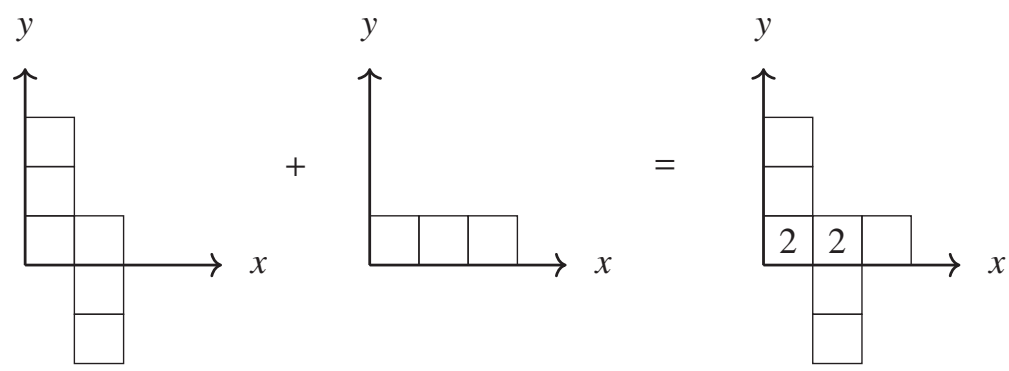

Figure 9. Quiver data for $(\square, \emptyset, \square) \in \operatorname{Hilb}\left(\mathcal{A}_{2}\right)$. 
Recall that an $m$-coloured partition $\lambda$ can also be described by its $m$-core $c(\lambda)$ and $m$-quotient $\left(q_{0}(\lambda), \ldots, q_{m-1}(\lambda)\right)$ (see [22, Section 2.7] for an introduction). Uniformly $m$-coloured partitions are precisely those with trivial $m$-core, so let

$$
\begin{aligned}
\boldsymbol{q}:\{\text { uniformly } m \text {-coloured partitions }\} & \stackrel{\sim}{\rightarrow}\{\text { all partitions }\}^{\times m} \\
\lambda & \mapsto\left(q_{0}(\lambda), \ldots, q_{m-1}(\lambda)\right)
\end{aligned}
$$

be the bijection.

Lemma 24 ([30, Proposition 2.5.3]). The bijection $\boldsymbol{q}$ is exactly the bijection

$$
\operatorname{Hilb}\left(\left[\mathbb{C}^{2} / \Gamma\right]\right)^{T^{\prime}} \cong \operatorname{Hilb}\left(\mathcal{A}_{m-1}\right)^{T^{\prime}}
$$

under our identification of fixed points with Young diagrams, and

$$
V_{k}(\lambda) \equiv V_{k}(\boldsymbol{q}(\lambda)) \bmod \hbar
$$

Importantly, (21) is only true mod $\hbar$ - that is, only in $K_{\mathrm{A}^{\prime}}(\mathrm{pt})-$ and is not true in $K_{\mathrm{T}^{\prime}}(\mathrm{pt})$. This means that $V(\lambda)$ and $V(\boldsymbol{q}(\lambda))$ may differ (and can only differ) by shifting boxes along the diagonals of the same colour. More precisely, to get the Young diagram represented by $V(\lambda)$ from $V(\boldsymbol{q}(\lambda)$ ), we shift boxes along their diagonals until we get a valid Young diagram, as in Figure 10.

As for obtaining $V(\boldsymbol{q}(\lambda))$ from $V(\lambda)$, the easiest way is somewhat indirect: Compute $\boldsymbol{q}(\lambda)$ directly from $V(\lambda)$ and then apply (20). It is not clear whether there is a nice combinatorial description of which boxes in $V(\lambda)$ need to be shifted (and by how much) to obtain $V(\boldsymbol{q}(\lambda))$ directly.

\subsection{7.}

There is a universal formula for the tangent space of both $\operatorname{Hilb}\left(\left[\mathbb{C}^{2} / \Gamma\right]\right)$ and $\operatorname{Hilb}\left(\mathcal{A}_{m-1}\right)$ at fixed points. This is because they are both open subsets of the stack $\left[\mu^{-1}(0) / G_{V}\right]$, whose tangent bundle in K-theory can be written as follows. If $V$ is the quiver data of a fixed point, then

$$
T_{V} \text { Hilb }=\overbrace{T^{*}\left(V_{0}+\frac{1}{x} \sum_{i=0}^{m-1} \operatorname{Hom}\left(V_{i}, V_{i+1}\right)\right)}^{\text {deformations of quiver rep }}-\overbrace{(1+\hbar) \sum_{i=0}^{m-1} \operatorname{Hom}\left(V_{i}, V_{i}\right)}^{\text {moment map and quotient }}
$$

Note that $T^{*} V_{i}=V_{i}+\hbar V_{i}^{\vee}$. The $x$ factor in front of $\operatorname{Hom}\left(V_{i}, V_{i+1}\right)$ is due to the multiplication-by- $x$ maps.
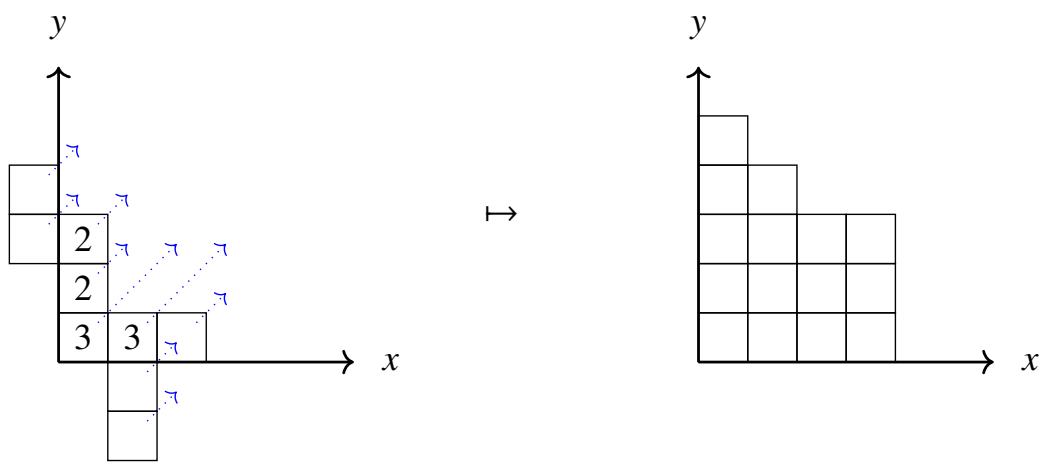

Figure 10. $T^{\prime}$-equivariant correspondence for $(\square, \square, \square) \in \operatorname{Hilb}\left(\mathcal{A}_{2}\right)$. 


\subsection{The quasimap vertex}

3.3.1.

To consider maps from a curve $C$ to a Nakajima quiver variety, we upgrade from quiver representations into vector spaces to quiver representations into vector bundles on $C$. Such objects are called quiver bundles [18].

Definition 25. For two vector bundles $\mathcal{V}=\bigoplus \mathcal{V}_{i}$ and $\mathcal{W}=\bigoplus \mathcal{W}_{i}$ on $C$, (10) can be upgraded to

$$
\mathcal{R e p}_{Q}^{\text {framed }}:=\bigoplus_{\text {edge } i \rightarrow j} \mathcal{H o m}\left(\mathcal{V}_{i}, \mathcal{V}_{j}\right) \oplus \bigoplus_{i \in I} \mathcal{H o m}\left(\mathcal{W}_{i}, \mathcal{V}_{i}\right)
$$

A section $s$ of $T^{*} \mathcal{R} \operatorname{ep}_{Q}^{\text {framed }}$ satisfying $\mu(s)=0$ is a quasimap in the sense of [9]; it is equivalent to a map

$$
f: C \rightarrow\left[\mu^{-1}(0) / G_{V}\right] .
$$

Because we want maps to the open locus $\mathcal{M}_{\boldsymbol{\theta}} \subset\left[\mu^{-1}(0) / G_{V}\right]$, we say a quasimap is singular at $p \in C$ if $f(p) \notin \mathcal{M}_{\boldsymbol{\theta}}$. If $f$ is singular only at finitely many points on $C$, it is a stable quasimap.

\subsection{2.}

Let $\mathrm{QMaps}\left(\mathcal{M}_{\boldsymbol{\theta}}\right)$ denote the moduli of stable quasimaps to the Nakajima quiver variety $\mathcal{M}_{\boldsymbol{\theta}}$ for $C=\mathbb{P}^{1}$. Consider the open locus

$$
\operatorname{QMaps}\left(\mathcal{M}_{\boldsymbol{\theta}}\right)_{\text {nonsing } \infty} \subset \operatorname{QMaps}\left(\mathcal{M}_{\boldsymbol{\theta}}\right)
$$

consisting of all quasimaps that are nonsingular at $\infty \in \mathbb{P}^{1}$. On this locus there is a well-defined evaluation map

$$
\mathrm{ev}_{\infty}: \operatorname{QMaps}\left(\mathcal{M}_{\boldsymbol{\theta}}\right)_{\text {nonsing } \infty} \rightarrow \mathcal{M}_{\boldsymbol{\theta}}
$$

sending $f$ to $\operatorname{ev}_{\infty}(f):=f(\infty)$.

Definition 26 ([36, Section 7.2]). The quasimap vertex is the series

$$
\mathrm{V}_{\mathrm{QMaps}}(\boldsymbol{z}):=\sum_{\boldsymbol{d}} \boldsymbol{z}^{\boldsymbol{d}} \mathrm{ev}_{\infty, *}\left(\mathrm{QMaps}^{\boldsymbol{d}}, \hat{\mathcal{O}}^{\mathrm{vir}}\right) \in K_{\mathrm{T}}\left(\mathcal{M}_{\boldsymbol{\theta}}\right)_{\text {localised }}\left[\left[\mathfrak{z}^{ \pm 1}\right]\right]
$$

The variables $\mathfrak{z}:=\left(\mathfrak{\jmath}_{0}, \ldots, \mathfrak{z} m-1\right)$ record the degree $\operatorname{deg} f:=\left(\operatorname{deg} \mathcal{V}_{i}\right) \in \mathbb{Z}^{m}$ of the quasimap, which indexes connected components $\operatorname{QMaps}^{\boldsymbol{d}} \subset \operatorname{QMaps}\left(\mathcal{M}_{\boldsymbol{\theta}}\right)_{\text {nonsing } \infty}$.

Remark 27. Quasimaps to Hilb $\left(\left[\mathbb{C}^{2} / \Gamma\right]\right)$ are studied modulo $\mathrm{A}^{\prime}$-equivariance in [12], where a formula for the quasimap vertex is given for the general $A_{\infty}$ quiver (which is a limiting case of affine $A_{n}$ quivers). The extension to full T-equivariance is straightforward. However, note that though the stability chamber (12) defining $\operatorname{Hilb}\left(\left[\mathbb{C}^{2} / \Gamma\right]\right)$ is valid on the $A_{\infty}$ quiver, the stability chamber (14) defining $\operatorname{Hilb}\left(\mathcal{A}_{m-1}\right)$ is not. It truly depends on $m$ and therefore cannot be investigated using the $A_{\infty}$ quiver.

\subsection{3.}

The domain $C=\mathbb{P}^{1}$ for quasimaps has an action by the $\mathbb{C}_{z}^{\times}$in $\mathrm{T}$ in (5) and corresponds to the $C$ in $Y=S \times C$. For $p \in \mathcal{M}_{\theta}$, let

$$
\mathrm{QMaps}_{p}:=\operatorname{ev}_{\infty}^{-1}(p)
$$

It has components QMaps ${ }_{p}^{\boldsymbol{d}}$ consisting of quasimaps of degree $\boldsymbol{d}$. It is known [36, Corollary 7.2.5] that the summands of

$$
\operatorname{QMaps}\left(\mathcal{M}_{\boldsymbol{\theta}}\right)_{\text {nonsing } \infty}^{\mathrm{T}}=\bigoplus_{p \in \mathcal{M}_{\boldsymbol{\theta}}^{\mathrm{T}}} \bigoplus_{\boldsymbol{d}}\left(\text { QMaps }_{p}^{\boldsymbol{d}}\right)^{\mathbb{C}_{z}^{\times}}
$$


are proper, and therefore the $p$ th component

$$
\mathrm{V}_{\mathrm{QMaps}}^{p}(\mathbf{z})=\sum_{\boldsymbol{d}} \chi\left(\mathrm{QMaps}_{p}^{\boldsymbol{d}}, \hat{\mathcal{O}}^{\mathrm{vir}}\right) \boldsymbol{z}^{\boldsymbol{d}}
$$

of the quasimap vertex is well defined via localisation.

\subsection{4.}

As with fixed points on the Nakajima quiver variety $\mathcal{M}_{\boldsymbol{\theta}}$, we conflate T-fixed quasimaps with their quiver data. Fix a point $V \in \mathcal{M}_{\boldsymbol{\theta}}^{\mathrm{T}^{\prime}}$, and let $f \in \mathrm{QMaps}_{V}^{\boldsymbol{d}}$ be a T-fixed quasimap defined by its quiver data $\mathcal{V}$.

Lemma 28 ([36, Section 7.1]). The map $f$ is constant, and $\mathcal{V}$ consists of line bundles $\mathcal{O}_{\mathbb{P}^{1}}(d)$ linearised with weights $z^{-d}, 1$ at $0, \infty \in \mathbb{P}^{1}$, respectively.

Definition 29. For a square $\square \in V$, let $d_{\llbracket}$ be the degree of its corresponding line bundle

$$
\mathcal{O}_{\mathbb{P}^{1}}\left(d_{\boxminus}\right) \subset \mathcal{V}
$$

If $\square$ has multiplicity - for example, for $\operatorname{Hilb}\left(\mathcal{A}_{m-1}\right)$ - then we write $d_{\square}^{(a)}$ for the degree coming from a $\in V^{(a)}(\lambda)$.

To represent $\mathcal{V}$ diagrammatically, we write (lists of) degrees in each box of the diagram for $V$, as in Figure 11. When there are multiple degrees in a box, we disregard the ordering of the list of degrees.

\subsection{5.}

There is a universal formula for the virtual tangent space of QMaps at $\mathcal{V}$. It is completely analogous to (22) but requires an extra pushforward over the domain $\mathbb{P}^{1}$ :

$$
T_{\mathcal{V}}^{\mathrm{vir} Q M a p s}=H^{\bullet}\left(T^{*}\left(\mathcal{V}_{0}+\frac{1}{x} \sum_{i=0}^{m-1} \operatorname{Hom}\left(\mathcal{V}_{i}, \mathcal{V}_{i+1}\right)\right)-(1+\hbar) \sum_{i=0}^{m-1} \operatorname{Hom}\left(\mathcal{V}_{i}, \mathcal{V}_{i}\right)\right)
$$

Usually for the quasimap vertex, one normalises by subtracting the contribution of $T_{V} \mathcal{M}_{\boldsymbol{\theta}}$ from $T_{\mathcal{V}}^{\mathrm{vir}}$. This is equivalent to replacing $H^{\bullet}$ in (24) with

$$
H_{\sim}^{\bullet}(\mathcal{O}(n)):=H^{\bullet}(\mathcal{O}(n))-1
$$

for nonequivariant line bundles $\mathcal{O}(n)$ on $\mathbb{P}^{1}$. The contribution $T_{V} \mathcal{M}_{\theta}$ is the freedom of the point $V$ to move around in $\mathcal{M}_{\theta}$, and removing it is analogous to the redistribution for the BS vertex discussed in section 2.2.4. Because we do not perform the redistribution for the BS vertex, we also do not normalise the quasimap vertex.

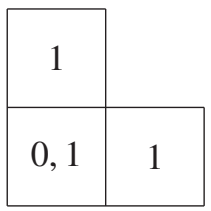

(a) Degree $(1,2)$ to $(\square, \square) \in \operatorname{Hilb}\left(\mathcal{A}_{1}\right)$

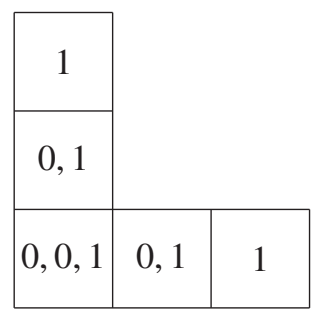

(b) Degree $(1,2,2)$ to $(\square, \square, \square) \in \operatorname{Hilb}\left(\mathcal{A}_{2}\right)$

Figure 11. Some T-fixed components of quasimap moduli space. 
3.3.6.

From here on, we focus on the case of quasimaps to $\operatorname{Hilb}\left(\mathcal{A}_{m-1}\right)$. Whereas the moduli of quasimaps to $\operatorname{Hilb}\left(\left[\mathbb{C}^{2} / \Gamma\right]\right)$ has isolated T-fixed points, the moduli of quasimaps to $\operatorname{Hilb}\left(\mathcal{A}_{m-1}\right)$ can have arbitrarily high-dimensional T-fixed loci. Using the formula (7) for $T^{\mathrm{vir}}$, one can read off a combinatorial formula for the virtual dimension of T-fixed loci.

Lemma 30. The T-fixed term in $T_{\mathcal{V}}^{v i r}$ is

1. the total number of pairs $d_{\square}^{(a)}>d^{(b)}$ where $\square^{\prime}$ is either $\square$ or $x y \cdot \square$, minus

2. the total number of pairs $d_{\square}^{(a)}>d_{\square^{\prime}}^{(b)}$ where $\square^{\prime}$ is either $x \cdot \square$ or $y \cdot \square$, minus

3. the total number of negative degrees at $\square=(0,0)$.

Proof. The T-fixed term is unchanged if we normalise $T_{\mathcal{V}}^{\mathrm{vir}}$ QMaps by using $H_{\sim}^{\bullet}$ as defined in (25) instead of the usual $H^{\bullet}$, because the discrepancy $T_{V} \mathcal{M}_{\theta}$ has no T-fixed weight. Using the formula (24) for $T_{\mathcal{V}}^{\mathrm{vir}}$, the T-fixed term is the total number of line bundles $\mathcal{O}_{\mathbb{P}^{1}}(n)$ with $n<0$ in the expression

$$
\mathcal{V}_{0}+\frac{\mathcal{V}_{0}^{\vee}}{x y}+\frac{1}{x} \sum_{a} \mathcal{V}_{a}^{\vee} \mathcal{V}_{a+1}+\frac{1}{y} \sum_{a} \mathcal{V}_{a} \mathcal{V}_{a+1}^{\vee}-\left(1+\frac{1}{x y}\right) \sum_{a} \mathcal{V}_{a}^{\vee} \mathcal{V}_{a}
$$

counted with opposite sign. Namely, a line bundle $\pm \mathcal{O}_{\mathbb{P}^{1}}(n)$ contributes $\mp 1$ to the total.

For example, the fixed component of Figure 11 has virtual dimension 4-2 = 2 in $\operatorname{QMaps}\left(\operatorname{Hilb}\left(\mathcal{A}_{2}\right)\right)$. Conjecturally, the fixed loci have sufficiently nice geometry for their virtual dimensions to be their actual dimensions.

Remark 31. Because $\pi$-stable pairs have no nontrivial automorphisms [5, Lemma 23], when the virtual dimension is negative there must be nontrivial obstructions and therefore the degrees do not define a valid quasimap fixed component. Hence, Lemma 30 yields a combinatorial criterion for how to label squares in $V(\lambda)$ to produce a valid quasimap.

\subsection{7.}

To understand the geometry of T-fixed loci in $\operatorname{QMaps}\left(\operatorname{Hilb}\left(\mathcal{A}_{m-1}\right)\right)$, a different perspective on T-fixed quasimaps is necessary.

Definition 32. Let $\mathcal{V} \in \operatorname{QMaps}\left(\operatorname{Hilb}\left(\mathcal{A}_{m-1}\right)\right)^{\mathrm{T}}$. Associated to $\mathcal{V}=\bigoplus_{k} \mathcal{V}_{k}$ are the vector spaces

$$
\mathbb{V}_{k}:=\bigoplus_{n \in \mathbb{Z}} \mathbb{V}_{k}[n]:=H^{0}\left(\left.\mathcal{V}_{k}\right|_{\mathbb{P}^{1} \backslash\{\infty\}}\right)
$$

where $\mathbb{V}_{k}[n]$ is the $\mathbb{C}_{z}^{\times}$weight space of weight $n$. Multiplication by $z$ induces embeddings

$$
\mathbb{V}_{k}[n] \hookrightarrow \mathbb{V}_{k}[n+1] \hookrightarrow \cdots \hookrightarrow \mathbb{V}_{k}[\infty]=V_{k}
$$

compatible with quiver maps. Let $\mathbb{V}^{\bullet}=\bigoplus_{k} \mathbb{V}_{k}^{\bullet}$ denote the resulting flag of quiver representations.

A T-fixed quasimap to $V$ is therefore the data of an infinite flag $\mathbb{V}^{\bullet}$ of (stable) quiver subrepresentations of $V$, starting with the zero quiver representation at $n=-\infty$ and ending with $V$ at $n=\infty$, along with the data of the framing morphism $\mathcal{W} \hookrightarrow \mathcal{V}$. Note that because $\mathcal{W}=\mathcal{O}_{\mathbb{P} 1}$, its associated flag is

$$
\mathbb{W}_{k}[n]= \begin{cases}W_{k} & n \geq 0 \\ 0 & n<0\end{cases}
$$

and there is a map $\mathbb{W}^{\bullet} \hookrightarrow \mathbb{V}^{\bullet}$. Rephrasing, the only obstruction to a flag $\mathbb{V}^{\bullet}$ of quiver subrepresentations of $V$ being a valid quasimap is that it admits a framing $\mathbb{W}^{\bullet} \hookrightarrow \mathbb{V}^{\bullet}$. 
Proposition 33. A quiver bundle $\mathcal{V}$ defined by a flag $\mathbb{V}^{\bullet}$ of quiver representations of $V$ is a stable quasimap iff it admits a framing $\mathbb{W}^{\bullet} \hookrightarrow \mathbb{V}^{\bullet}$.

3.3.8.

Using $\mathbb{V}^{\bullet}$, we now explain the ambiguity in defining a single quasimap from diagrams like in Figure 11. The problem is that, even after specifying the dimension vector for a quiver subrepresentation, there is still nontrivial moduli for the quiver subrepresentation whenever a square has multiplicity. This occurs in negative z-degree, where, crucially, the subrepresentations are not framed. Note that this will only occur for QMaps $\left(\operatorname{Hilb}\left(\mathcal{A}_{m-1}\right)\right)$; the T-fixed loci in $\operatorname{QMaps}\left(\operatorname{Hilb}\left(\left[\mathbb{C}^{2} / \Gamma\right]\right)\right)$ are isolated points.

Example 34. Consider the T-fixed component in Figure 11. Disregarding $z$-weights, it corresponds to the flag

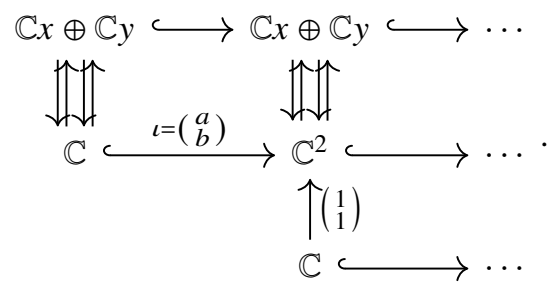

There is a $\mathbb{P}^{1}$ worth of freedom for the inclusion $\iota: \mathbb{C} \rightarrow \mathbb{C}^{2}$, and then everything else is uniquely specified. Hence, this T-fixed component is a $\mathbb{P}^{1}$.

This example is the quasimap analogue of Example 14. Note that if the $\mathbb{V}[-1]$ slice were also framed, the framing would force $\iota=\left(\begin{array}{l}1 \\ 1\end{array}\right)$, removing the degree of freedom and turning the fixed $\mathbb{P}^{1}$ into a fixed point.

\section{BS/quasimaps correspondence}

\subsection{The correspondence}

\subsection{1.}

The main result of this article is that, after a suitable change of variables, the series in the BS 1-leg vertex and the quasimap vertex for the $\mathcal{A}_{m-1}$ geometry are equal on the nose.

Theorem 35 (BS/quasimaps correspondence). For $S=\mathcal{A}_{m-1}$,

$$
V_{\pi-P a i r s\left(S \times \mathbb{P}^{1}\right)}(Q, A)=V_{Q M a p s(\operatorname{Hilb}(S))}(\mathfrak{z})
$$

after the change of variables

$$
\begin{aligned}
Q & \leftrightarrow z_{0} z_{1} \cdots z_{m-1} \\
A_{i} & \leftrightarrow z_{i} \quad \forall 1 \leq i<m .
\end{aligned}
$$

As with many results about $\mathcal{A}_{m-1}$, this theorem continues to hold when $m=1$. In this case, the geometry is $\mathcal{A}_{0}:=\mathbb{C}^{2}$, viewed as a trivial resolution of $\mathbb{C}^{2}$ with no singularities. Then BS theory on $\mathcal{A}_{0}$ is equivalent to PT theory, in which case this theorem shows that

$$
\mathrm{V}_{\mathrm{Pairs}\left(\mathbb{C}^{2}\right)}(Q)=\mathrm{V}_{\mathrm{QMaps}\left(\operatorname{Hilb}\left(\mathbb{C}^{2}\right)\right)}(\mathfrak{3})
$$

where $Q=\mathfrak{z}$. In fact, in this case, this arises from a known isomorphism Pairs $\left(\mathbb{C}^{2}\right) \simeq \operatorname{QMaps}\left(\operatorname{Hilb}\left(\mathbb{C}^{2}\right)\right)$ of moduli spaces [36, Exercise 4.3.2]. 


\subsection{2.}

The proof of the correspondence goes via T-equivariant localisation: It suffices to give an isomorphism of T-fixed loci for $\pi$-Pairs and QMaps and show that this isomorphism respects virtual tangent spaces. We first state this isomorphism as a bijection between components of T-fixed loci and then clarify why it is an isomorphism. Fix $\lambda \in \operatorname{Hilb}\left(\mathcal{A}_{m-1}\right)^{\mathrm{T}}$.

- Recall from Subsection 2.3.11 that T-fixed (components of) $\pi$-stable pairs to $\lambda$ are specified by local models for each $\square \in \lambda_{a}$. In particular, the local model for $\square \in \lambda_{a}$ is defined by numbers

$$
\boldsymbol{e}^{\square}:=\left(e_{0}^{\square}, \ldots, e_{m-1}^{\square}\right) .
$$

Here $e_{0}^{\square}$ is the smallest integer $k \in \mathbb{Z}$ such that the box $z^{k} \cdot \square$ is not involved in a rod, and otherwise $e_{a}^{\square}$ is the integer such that $e_{a}^{\square}-e_{0}^{\square}$ is the number of standard rods in the local model with nonzero support over the exceptional component $E_{a}$.

○ Recall from Subsection 3.3.4 that if $V(\lambda)=\sum_{a} V^{(a)}(\lambda)$ is the quiver data describing $\lambda$, then Tfixed (components of) quasimaps to $\lambda$ are specified by labelling the squares in each $V^{(a)}(\lambda)$ with degrees. More specifically, each $\square \in \lambda_{a}$ has an associated sequence of degrees

$$
\boldsymbol{d}^{\square}:=\left(d_{0}^{\square}, \ldots, d_{m-1}^{\square}\right),
$$

namely, where $d_{a}^{\square}$ is the label of the colour- $a$ square in the hook with character $\mathrm{hk}_{a} x_{a}^{i(\square)} y_{a}^{j(\square)} \subset$ $V^{(a)}(\lambda)$.

The desired bijection is simply that

$$
\boldsymbol{e}^{\square}=\boldsymbol{d}^{\square} \quad \forall \square \in \lambda_{a}, \forall 0 \leq a<m .
$$

That this is indeed a bijection of fixed components is the content of Proposition 42, obtained by manually matching stability conditions in BS and quasimap theories. One can easily check that this bijection yields the specified change of variables $(Q, \boldsymbol{A}) \leftrightarrow \mathfrak{z}$.

\subsection{3.}

The outline of the proof is as follows. In Subsection 4.2, the bijection $\boldsymbol{d}^{\square} \mapsto \boldsymbol{e}^{\square}$ is realised geometrically as an equivalence of categories

$$
\widetilde{\Phi}_{\mathrm{T}}: D^{b} \operatorname{Coh}_{\mathrm{T}}\left(\mathbb{C}^{2} \times \mathbb{P}^{1}\right) \rightarrow D^{b} \operatorname{Coh}_{\mathrm{T} / \Gamma}\left(\mathcal{A}_{m-1} \times \mathbb{P}^{1}\right),
$$

constructed using a T-equivariant version of the derived McKay equivalence. Consequently, the bijection of fixed components is refined to an isomorphism of fixed components, discussed in Subsection 4.2.6. Then, to match vertices, it suffices to match virtual tangent spaces of fixed components. Although not all $\left\{\boldsymbol{d}^{\square}\right\}_{\square \in \lambda}$ form stable quasimaps, and not all $\left\{\boldsymbol{e}^{\square}\right\}_{\square \in \lambda}$ form valid $\mathcal{O}_{Y}$-modules for $\pi$-stable pairs, in fact Proposition 39 shows that the formulas (7) and (24) for their virtual tangent spaces agree for any $\boldsymbol{d}^{\square}=\boldsymbol{e}^{\square}$ via $\widetilde{\Psi}_{\mathrm{T}}$.

For the equality of BS and quasimap vertices, it suffices to show that $\widetilde{\Phi}_{\mathrm{T}}$ sends stable T-fixed quasimaps to valid T-fixed $\pi$-stable pairs. This is done in Subsection 4.3. However, it is in fact true that $\widetilde{\Phi}_{\mathrm{T}}$ sends all stable quasimaps to valid $\pi$-stable pairs: Proposition 45 shows that it provides a T-equivariant isomorphism of moduli spaces, not just of T-fixed loci.

\subsection{Equivariant derived McKay}

\subsection{1.}

When $Y \rightarrow \mathfrak{V}$ is a crepant resolution of a sufficiently nice orbifold singularity, it is generally expected that $D^{b} \operatorname{Coh}(Y)$ and $D^{b} \operatorname{Coh}(\mathfrak{Y})$ are equivalent. Historically, this was first proved for ADE surfaces $S$, 
which are crepant resolutions of the surface singularities $\left[\mathbb{C}^{2} / \Gamma\right]$. The equivalence of categories is given by a Fourier-Mukai transform, whose kernel is the incidence correspondence

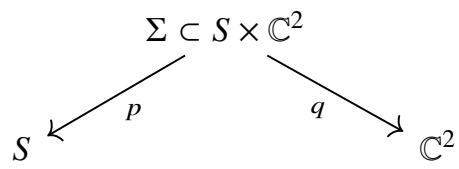

given by viewing points on $S$ as $\Gamma$-orbits of $m$ points in $\mathbb{C}^{2}$. Later this was generalised to crepant resolutions of threefold singularities $[M / \Gamma]$ for affine $M[3]$.

Theorem 36 ([3, Theorem 1.1]). The Fourier-Mukai transform

$$
\Psi(-):=R q_{*}\left(\mathcal{O}_{\Sigma} \otimes p^{*}\left(-\otimes R_{0}\right)\right): D^{b} \operatorname{Coh}(S) \rightarrow D^{b} \operatorname{Coh}_{\Gamma}\left(\mathbb{C}^{2}\right)
$$

is an equivalence of categories.

Although in our setting $Y=\mathcal{A}_{m-1} \times C$ is a 3 -fold, it is easier to understand $\Psi$ for the surface case and then use the equivalence relatively over $C$. At the level of equivariant K-theory, $\Psi$ induces an isomorphism

$$
\Psi: K(S) \cong K_{\Gamma}\left(\mathbb{C}^{2}\right)
$$

known earlier to Gonzalez-Sprinberg and Verdier [17], which provides a geometric explanation for the classical McKay correspondence. As such, the equivalence $\Psi$ is known as the derived McKay correspondence. We use $\Psi$ to denote both the derived equivalence and the K-theoretic isomorphism.

\subsection{2.}

The first step in the proof of the BS/quasimaps correspondence is to match the virtual tangent sheaves $T^{\mathrm{vir}}$ on both sides, which is the content of Proposition 39. This requires us to compute that the bijection of Subsection 4.1 .2 between T-fixed $\pi$-stable pairs and quasimaps is just an application of the equivalence $\Psi$. The isomorphism of K-theories then yields a comparison of fibres of $T^{\mathrm{vir}}$.

Because the $T^{\mathrm{vir}}$ are T-equivariant objects, it is important to extend the equivalence $\Psi$ to the fully T-equivariant

$$
\Psi_{\mathrm{T}}: D^{b} \operatorname{Coh}_{\mathrm{T} / \Gamma}(S) \rightarrow D^{b} \operatorname{Coh}_{\mathrm{T}}\left(\mathbb{C}^{2}\right),
$$

which we call the equivariant derived McKay equivalence. It is straightforward but slightly tedious to check that all machinery used in the proof [3, Section 6] of Theorem 36 holds in the T-equivariant setting as well. Note that this is not automatic; a similar correspondence with Fourier-Mukai kernel $\mathcal{O}_{\Sigma}^{\vee}$ instead of $\mathcal{O}_{\Sigma}$ is defined in [23] and shown to be an equivalence $D^{b} \operatorname{Coh}(S) \simeq D^{b} \operatorname{Coh}_{\Gamma}\left(\mathbb{C}^{2}\right)$, but it does not extend to a T-equivariant equivalence.

4.2.3.

By the general theory of Fourier-Mukai transforms, the inverse of $\Psi_{\mathrm{T}}$ must be its left (and right) adjoint

$$
\Phi_{\mathrm{T}}(-):=\left[R p_{*}\left(\mathcal{P} \otimes q^{*}(-)\right)\right]^{\Gamma}: D^{b} \operatorname{Coh}_{\mathrm{T}}\left(\mathbb{C}^{2}\right) \rightarrow D^{b} \operatorname{Coh}_{\mathrm{T} / \Gamma}(S)
$$

where, T-equivariantly,

$$
\mathcal{P}:=\mathcal{O}_{\Sigma}^{\vee} \otimes q^{*}\left(\omega_{\mathbb{C}^{2}}\right)[2]=x y \mathcal{O}_{\Sigma}^{\vee}[2]
$$

Let $\iota:\{0\} \rightarrow \mathbb{C}^{2}$ be the inclusion. The crucial computation identifying quasimap data with $\pi$-stable pair data is the image of skyscrapers $\iota_{*} w:=w \cdot \iota_{*} \mathcal{O}_{0}$ under $\Phi_{\mathrm{T}}$, for weights $w \in K_{T}(\mathrm{pt})$. Note that $\Phi_{\mathrm{T}}$ is $\mathrm{T} / \Gamma$-linear, namely,

$$
\Phi_{\mathrm{T}}\left(w_{0} \mathcal{E}\right)=w_{0} \Phi_{\mathrm{T}}(\mathcal{E}) \quad \forall w_{0} \in K_{\mathrm{T} / \Gamma}(\mathrm{pt}) .
$$


Hence, for $S=\mathcal{A}_{m-1}$, it suffices to compute $\Phi\left(\iota_{*} x^{k}\right)$ for $0 \leq k<m$. There are many ways to do so - for example, using the same process as in [23, Section 2] - but because at the end of the day we work only in K-theory, it is instructive and fairly straightforward to do the calculation there and then lift the result up to $D^{b}$ Coh using some interesting general theory.

Lemma 37. For $S=\mathcal{A}_{m-1}$,

$$
\Phi_{T}\left(\iota_{*} x^{k}\right)= \begin{cases}\mathcal{O}_{E}(-2)[1] & k=0 \\ \mathcal{O}_{E_{k}}(-1) & \text { otherwise }\end{cases}
$$

where the T-linearisations are given by

$$
\begin{gathered}
\left.\mathcal{O}_{E}(-2)\right|_{p_{0}}=x_{0} \\
\left.\mathcal{O}_{E_{k}}(-1)\right|_{p_{k}}=x_{k}
\end{gathered}
$$

Proof. A priori $\Phi_{\mathrm{T}}\left(\iota_{*} x^{k}\right)$ is a complex of sheaves, but [7, Theorem 1.1] shows that it is actually some shift of a coherent sheaf. Computing $\Phi_{\mathrm{T}}\left(\iota_{*} x^{k}\right)$ in equivariant K-theory identifies the sheaf, and then cohomological considerations identify the shift. Apply the equivariant Koszul resolution of $\mathcal{O}_{0}$ simultaneously to all fibres of $\mathcal{O}_{\Sigma}$ and dualise to get the equivariant resolution

$$
x y p^{*} V^{\vee} \rightarrow x p^{*} V^{\vee} \oplus y p^{*} V^{\vee} \rightarrow p^{*} V^{\vee}
$$

of $x y \mathcal{O}_{\Sigma}^{\vee}$. Here $V=\bigoplus_{k=0}^{m-1} V_{k}$ is the universal family on $\mathcal{A}_{m-1}$ described in Subsection 3.2.4; equivalently, $V=p_{*} \mathcal{O}_{\Sigma}$. By push-pull, it follows that

$$
\Phi_{\mathrm{T}}\left(\iota_{*} x^{k}\right)=x^{k} \cdot\left[x y V_{k}^{\vee} \rightarrow x V_{k+1}^{\vee} \oplus y V_{k-1}^{\vee} \rightarrow V_{k}^{\vee}\right] \in D^{b} \operatorname{Coh}_{\mathrm{T} / \Gamma}\left(\mathcal{A}_{m-1}\right),
$$

where indices should be taken modulo $m$. It is now easy to identify the resulting sheaf by computing its K-theoretic weight in each chart or at each fixed point, using (17). For example, in an appropriate localisation of $K_{\mathrm{T} / \Gamma}\left(U_{a}\right)$, the modules

$$
\Gamma\left(U_{a}, \Phi_{\mathrm{T}}\left(\iota_{*} 1\right)\right)=\frac{1}{\left(1-x_{a}\right)\left(1-y_{a}\right)} \cdot \begin{cases}x y-x / y^{m-1} & a=0 \\ x y-1 & 1 \leq a<m-1 \\ x y-y / x^{m-1} & a=m-1\end{cases}
$$

glue to form $-\mathcal{O}_{E}(-2)$ with the desired linearisation. (It is helpful to visualise this by drawing the appropriate box configuration in each chart.) Finally, [7, Section 5] actually shows that (28) has cohomological support in degrees $[-2,0]$ when $k=0$ and in degrees $[-1,0]$ when $k \neq 0$, which uniquely determines the cohomological shift of the resulting sheaves for both cases.

\subsection{4.}

It remains to lift $\Phi_{\mathrm{T}}$ to the 3 -fold setting of $Y=\mathcal{A}_{m-1} \times \mathbb{P}^{1}$. Let $X:=\mathbb{C}^{2} \times \mathbb{P}^{1}$. Note that performing $\Phi_{\mathrm{T}}$ on fibres of $X$ relative to $\mathbb{P}^{1}$ induces an equivalence

$$
\widetilde{\Phi}_{\mathrm{T}}: D^{b} \operatorname{Coh}_{\mathrm{T}}(X) \rightarrow D^{b} \operatorname{Coh}_{\mathrm{T} / \Gamma}(Y)
$$

Let $\iota:\{0\} \times \mathbb{P}^{1} \hookrightarrow X$ be the inclusion. Given a T-fixed quasimap $\mathcal{V} \in$ QMaps $_{V}$, view it as a flag of quiver subrepresentations $\mathbb{V}[n] \subset V$ as in (26), where the flag inclusions are multiplication-by- $z$ maps. Then, by definition, $\widetilde{\Phi}_{\mathrm{T}}\left(\iota_{*} \mathcal{V}\right)$ is constructed from the pieces $\Phi_{\mathrm{T}}\left(\iota_{*} \mathbb{V}[n]\right)$ with $z$-weight $n$, linked together by the induced multiplication-by- $z$ maps.

Lemma 38. The bijection of Subsection 4.1.2 is exactly $\widetilde{\Phi}_{T}$ in K-theory. 
Proof. From the discussion above and the $T / \Gamma$-linearity of $\widetilde{\Phi}_{\mathrm{T}}$, it suffices to compute the image of the hook $\mathrm{hk}_{a}$ labelled with the degrees $\boldsymbol{d}^{\square}$ and show that the result is the local model with data $\boldsymbol{e}^{\square}=\boldsymbol{d}^{\square}$. Here $\mathrm{hk}_{a}$ is the $m$-hook corresponding to the $a$ th leg, from (19). Using the calculation of Lemma 37:

- $\Phi_{\mathrm{T}}\left(\iota_{*} \mathrm{hk}_{a}\right)$ is a single box 四 $\in U_{a}$ of weight 1 ;

- $\Phi_{\mathrm{T}}\left(\iota_{*}\left(x^{a-k}+\cdots+x^{a}\right)\right)$ is a standard leftward rod of length $k$ from $⿴$;

○ $\Phi_{\mathrm{T}}\left(\iota_{*}\left(y^{a-k}+\cdots+y^{a}\right)\right)$ is a standard rightward rod of length $k$ from 四. Here we are using that $\Phi_{\mathrm{T}}\left(\iota_{*} y^{m-j}\right)=y_{j} \Phi_{\mathrm{T}}\left(\iota_{*} x^{j}\right)$.

Attaching these pieces together with the natural multiplication-by- $z$ maps yields the desired local model.

\subsection{5.}

Let $I^{\bullet}=\left[\mathcal{O}_{Y} \rightarrow \mathcal{F}\right]$ be a T-fixed $\pi$-stable pair with corresponding T-fixed quasimap $\mathcal{V}$. In this notation, Lemma 38 shows that

$$
\mathcal{F}=\widetilde{\Phi}_{\mathrm{T}}\left(\iota_{*} \mathcal{V}\right) \in K_{\mathrm{T} / \Gamma}(Y)
$$

It is easy to verify that $\widetilde{\Phi}_{\mathrm{T}}\left(\mathcal{O}_{X}\right)=\mathcal{O}_{Y}$. Because $I^{\bullet}=\mathcal{O}_{Y}-\mathcal{F}$, it follows that

$$
I^{\bullet}=\widetilde{\Phi}_{\mathrm{T}}\left(I_{\mathcal{V}}\right),
$$

where $I_{\mathcal{V}}:=\mathcal{O}_{X}-i_{*} \mathcal{V}$.

Proposition 39. In $K_{T / \Gamma}(p t)$,

$$
T_{\left[I^{\bullet}\right]}^{v i r} \pi \text {-Pairs }=T_{\mathcal{V}}^{v i r} \text { QMaps }
$$

Proof. By the local-to-global Ext spectral sequence,

$$
T_{\left[I^{\bullet}\right]}^{\mathrm{vir}}=H_{Y}^{\bullet}\left(\mathcal{E} \mathrm{xt}_{Y}^{\bullet}\left(\mathcal{O}_{Y}, \mathcal{O}_{Y}\right)-\mathcal{E x t}_{Y}^{\bullet}\left(I^{\bullet}, I^{\bullet}\right)\right) .
$$

Using that $\widetilde{\Phi}_{\mathrm{T}}$ is an equivalence of categories,

$$
T_{\left[I^{\bullet}\right]}^{\mathrm{vir}}=H_{X}^{\bullet}\left(\mathcal{E x t}_{X}^{\bullet}\left(\mathcal{O}_{X}, \mathcal{O}_{X}\right)-\mathcal{E} \mathrm{xt}_{X}^{\bullet}\left(I_{\mathcal{V}}, I_{\mathcal{V}}\right)\right)^{\Gamma}
$$

The quantity in the right-hand side that $H_{X}^{\bullet}$ is applied to is a sheafy version of $T_{V} \operatorname{Hilb}\left(\mathbb{C}^{2}\right)$, for which the usual formula applies (see, e.g., [27, Section 4.7]):

$$
\mathcal{E x t}_{X}^{\bullet}\left(\mathcal{O}_{X}, \mathcal{O}_{X}\right)-\mathcal{E x t}_{X}^{\bullet}\left(I_{\mathcal{V}}, I_{\mathcal{V}}\right)=\iota_{*}\left(\mathcal{V}+\frac{\mathcal{V}^{\vee}}{x y}-\mathcal{V} \mathcal{V}^{\vee} \frac{(1-x)(1-y)}{x y}\right)
$$

Use $H_{X}^{\bullet} \circ \iota_{*}=H_{\mathbb{P} 1}^{\bullet}$ to simplify. Because $(-)^{\Gamma}$ commutes with $H_{\mathbb{P} 1}^{\bullet}$, we can take $\Gamma$-invariants first. But $\Gamma$ invariants of (29) yield exactly a sheafy version of $T_{V} \operatorname{Hilb}\left(\mathcal{A}_{m-1}\right)$. Then taking $H_{\mathbb{P} 1}^{\bullet}$ gives $T_{\mathcal{V}}^{\mathrm{vir}} \mathrm{QMaps}$, as in (24).

4.2.6.

Although the description of $\widetilde{\Phi}_{\mathrm{T}}$ so far is as a bijection between T-fixed components of $\pi$-stable pairs and quasimaps, it naturally refines into an isomorphism of fixed components as follows. The description of $\Phi_{\mathrm{T}}$ in Lemma 37 should be viewed as a correspondence between individual squares in each $\mathbb{V}[n]$ of a quasimap and individual standard rods in $\mathcal{F}$ of a $\pi$-stable pair. The remaining data on either side are how these individual pieces attach or glue to each other, via either quiver maps or $\mathcal{O}_{Y}$-module maps. Hence, it remains to match the gluing data on both sides. 
Example 40. On $\mathcal{A}_{1}$, remembering the quiver maps from colour-0 squares (to non-colour-0 squares) as $\widetilde{\Phi}_{\mathrm{T}}$ is applied yields, for example,

$$
b \underbrace{}_{a} \sim\left[\mathcal{O}_{E}(-2) \stackrel{\left(\begin{array}{l}
a \\
b
\end{array}\right)}{\longrightarrow} \mathcal{O}_{E}(-1) \oplus \mathcal{O}_{E}(-1) y / x\right],
$$

where the linearisations on the sheaves are the ones in Lemma 37. If both $a$ and $b$ are nonzero, then the resulting complex is quasi-isomorphic to the $\mathcal{O}_{Y}$-module $\mathcal{O}_{E}$, whereas if only one is zero, then the result is (nonequivariantly) $\mathcal{O}_{\mathrm{pt}} \oplus \mathcal{O}_{E}(-1)$. This matches the degrees of freedom (for a $\mathbb{P}^{1}$ fixed component) in Example 14 and Example 34.

The above example is representative of the general case, at any $\mathcal{A}_{1}$ piece inside $\mathcal{A}_{m-1}$, for how quiver maps from colour- 0 squares correspond to $\mathcal{O}_{Y}$-module maps involving certain boxes/rods.

For quiver maps from non-colour-0 squares, the $\mathcal{O}_{Y}$-module maps that correspond to each quiver map are described implicitly in the proof of Lemma 43. Split each $V^{(a)}(\lambda)$ into its hooks. The quiver maps between squares from the same hook correspond to $\mathcal{O}_{Y}$-module maps within each local model prescribing how standard rods are attached to each other. The quiver maps between squares from different hooks correspond to maps from white to gray boxes in Figures 12 and 13.

Remark 41. After localisation to fixed components, the vertex on either side is computed by a further integration $\chi\left(F, \hat{\mathcal{O}}^{\text {vir }}\right)$ over each fixed component $F$. The isomorphism of fixed components along with the equality of $T^{\mathrm{vir}}$ given by Proposition 39 implies the equality of $\chi\left(F, \hat{\mathcal{O}}^{\mathrm{vir}}\right)$ in BS and quasimap theories.

\subsection{Stability conditions}

4.3.1.

It remains to show that $F \subset \pi$-Pairs ${ }_{\lambda}^{\mathrm{T}}$ iff $\widetilde{\Phi}_{\mathrm{T}}(F) \subset \mathrm{QMaps}_{\lambda}^{\mathrm{T}}$. The idea is to identify two combinatorial conditions on the degrees

$$
\left\{d_{\square} \mid \square \in V(\lambda)\right\}
$$

and then to show that on the BS side they are equivalent to the corresponding $\mathcal{F}$ forming a $\pi$-stable pair and on the quasimap side they are equivalent to the corresponding $\mathcal{V}$ forming a stable quasimap.

Proposition 42. The degrees

$$
\left\{d_{\square} \mid \square \in V(\lambda)\right\}
$$

define a $\pi$-stable pair $\left[\mathcal{O}_{Y} \rightarrow \mathcal{F}\right] \in \pi$-Pairs ${ }_{V}$ iff they define a stable quasimap $\mathcal{V} \in$ QMaps $_{V}$.

Proof. It will follow from Lemma 43 that $\mathcal{F}$ is a valid $\mathcal{O}_{Y}$-module iff $\mathcal{V}$ is a valid quiver bundle. Proposition 13 shows that a sheaf $\mathcal{F}$ built from local models is a $\pi$-stable pair iff it admits an inclusion $\mathcal{O}_{C} \hookrightarrow \mathcal{F}$. Similarly, Proposition 33 shows that a quiver bundle $\mathcal{V}$ is a stable quasimap iff it admits a framing $\mathcal{W} \hookrightarrow \mathcal{V}$. Then, to conclude, Lemma 44 will show that the quiver bundle $\mathcal{V}$ admits an inclusion from $\mathcal{W}=\mathcal{O}_{\mathbb{P}^{1}}$ iff $\mathcal{F}$ admits an inclusion from $\mathcal{O}_{C}$.

\subsection{2.}

Let $\lambda \in \operatorname{Hilb}\left(\mathcal{A}_{m-1}\right)^{\mathrm{T}^{\prime}}$ and consider the labelling of $V(\lambda)=\sum_{a} V^{(a)}(\lambda)$ by the degrees $\left\{d_{\square}\right\}$. By viewing quiver bundles as flags of quiver representations or otherwise, it is clear that in order for this labelling to produce a valid quiver bundle $\mathcal{V}$, the degrees of boxes in each $V^{(a)}$ must satisfy the property

$$
d_{\square} \leq d_{x \cdot \boxminus}, d_{y \cdot \boxminus \quad \forall \square \in V^{(a)}}
$$


whenever the squares $x \cdot \square$ and $y \cdot \square$ exist in $V^{(a)}$. There is an ambiguity in identifying which degrees are for which $V^{(a)}$ at squares with multiplicity, but in order for the resulting $\mathcal{V}$ to form a valid quiver bundle there must be at least one identification satisfying property (31). This is analogous to picking the filtration for BS local models in Proposition 12. Hence, in what follows, it suffices to work with a single $V^{(a)}$. Equivalently, we assume that $\lambda$ is empty except at $\lambda_{a} \neq \emptyset$, so that $V=V^{(a)}$.

Lemma 43. The degrees

$$
\left\{d_{\square} \mid \square \in V^{(a)}\right\}
$$

define a valid $\mathcal{O}_{Y}$-module $\mathcal{F}^{(a)}$ iff they satisfy the condition (31).

Proof. It is easier to group the squares in $V^{(a)}$ into the hooks associated to each $\square \in \lambda_{a}$ and work with the degrees $\left\{\boldsymbol{d}^{\square}\right\}_{\square \in \lambda_{a}}$ instead of the degrees $\left\{d_{\square}\right\}_{\square \in V^{(a)}}$. Then to check condition (31) using $\left\{\boldsymbol{d}^{\square}\right\}_{\square \in \lambda_{a}}$, it is equivalent to check that it holds for the squares in each individual hook and then check that it holds for adjacent squares coming from two different hooks. For example, consider the hook in $V^{(a)}$ associated to $\square \in \lambda_{a}$, whose squares have degrees $\boldsymbol{d}^{\square}$. From the discussion of Subsection 2.3.6, it is clear that the local model given by the single hook forms a valid $\mathcal{O}_{Y}$-module iff the degrees of the hook satisfy (31).

Now consider two different hooks given by $\square$ and $\square^{\prime}:=x_{a} \cdot \square$ whenever both are in $\lambda_{a}$. The corresponding hooks in $V^{(a)}$ are adjacent at exactly one location, and the desired inequality is

$$
d_{a+1}^{\square^{\prime}} \geq d_{a}^{\square}
$$

The relevant portion of the local models is shown in Figure 12, where boxes in the local model for $\square^{\prime}$ are coloured gray. Suppose 四:= $z^{k} \cdot \square$ generates a standard leftward rod. Because the $\square^{\prime}$ column exists in $L_{a}$, it must also generate a box 四' $:=x_{a}$. 目. This box 四' must be part of the local model associated to $\square^{\prime}$. Moreover, 四' cannot generate a standard leftward rod; otherwise, there is no nonzero $\mathcal{O}_{Y}$-module map $x_{a}$ : 四 $\rightarrow$ 四'. Hence, 四' generates a standard rightward rod, and the desired inequality follows. Note that an analogous argument can be given for $\square$ and $\square^{\prime}:=y_{a} \cdot \square$, as shown in Figure 12, where the desired inequality is $d_{a}^{\square^{\prime}} \geq d_{a+1}^{\square}$.

Equivalently, using the notation of Subsection 2.3.9, the inequality follows from the nonzero $\mathcal{O}_{Y^{-}}$ module maps of $\mathcal{F}^{z}$ between adjacent columns. These multiplication-by- $x_{a}$ or $y_{a}$ maps are nonzero because they are nonzero in $L_{a}$, and all boxes in $\mathcal{F}^{z}$ eventually generate $L_{a}$.

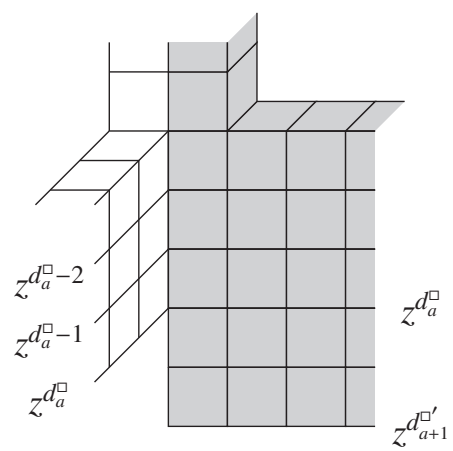

(a) Multiplication by $x_{a}$

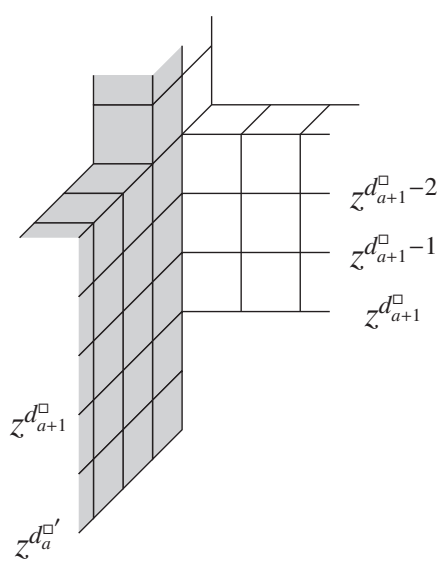

(b) Multiplication by $y_{a}$

Figure 12. Portions of local models associated to adjacent columns. 
Finally, consider $\square$ and $\square^{\prime}:=x y \cdot \square$. This is the only remaining case where the corresponding hooks have adjacent squares in $V^{(a)}$. Again, nonzero $\mathcal{O}_{Y}$-module maps in $\mathcal{F}^{z}$ yield the inequalities

$$
d_{0}^{\square^{\prime}} \geq d_{m-1}^{\square}, d_{1}^{\square}
$$

(When $a=0$ or $a=m-1$, only the appropriate one applies.) It remains to explain the inequalities

$$
\begin{array}{ll}
d_{b}^{\square^{\prime}} \geq d_{b-1}^{\square} & \forall a<b<m-1 \\
d_{b}^{\square^{\prime}} \geq d_{b+1}^{\square} & \forall 0<b \leq a .
\end{array}
$$

These arise from the behaviour of standard rods in the $\mathcal{O} \oplus \mathcal{O}(-2)$ geometry. Namely, if both boxes 四:= $z^{k} \cdot \square$ and $x y \cdot$ 四 generate standard rightward rods $\mathcal{R}$ and $\mathcal{R}^{\prime}$ of length $\ell$ and $\ell^{\prime}$, it must be that $\ell^{\prime}>\ell$. This is because the rightmost box of $\mathcal{R}$, of weight $y_{a+\ell} \cdot$ 四, must generate in the $x_{a+\ell}$ direction another box of weight

$$
x_{a+\ell} y_{a+\ell} \cdot \text { 四 }=x y \cdot \text { 四, }
$$

which must belong to $\mathcal{R}^{\prime}$. If $\mathcal{R}^{\prime}$ were of length $\ell$, its rightmost box would be $y_{a+\ell} x y \cdot$ 四, a contradiction. This is shown in Figure 13, where $\mathcal{R}^{\prime}$ is in gray. Hence, $\ell^{\prime}>\ell$, which yields (32a). The analogous argument holds for standard leftward rods, shown in Figure 13, and yields (32b).

We have just shown that every $\mathcal{O}_{Y}$-module $\mathcal{F}^{(a)}$ with one nontrivial external leg formed from local models yields quasimap degrees $\left\{d_{\llbracket}\right\}_{\curvearrowleft \in V^{(a)}}$ satisfying (31) by verifying the necessary inequalities. Conversely, it is clear that these inequalities are sufficient to create a valid $\mathcal{O}_{Y}$-module from local models.

\subsection{3.}

Within $\mathcal{F}=\widetilde{\Phi}_{\mathrm{T}}\left(\iota_{*} \mathcal{V}\right)$ must be a unique subsheaf $\mathcal{O}_{C}$ that is the structure sheaf of a CM curve containing the infinite legs $L_{a}$. To finish the proof of Proposition 42, we need to identify the conditions on $\mathcal{V}$ in order for there to be an inclusion $\mathcal{O}_{C} \hookrightarrow \mathcal{F}$.

Lemma 44. Let $\mathcal{F}=\widetilde{\Phi}_{T}\left(\iota_{*} \mathcal{V}\right)$ be an $\mathcal{O}_{Y}$-module. Then $\mathcal{F}$ admits an inclusion $\mathcal{O}_{C} \hookrightarrow \mathcal{F}$ iff $\mathcal{V}$ admits a framing $\mathcal{W} \hookrightarrow \mathcal{V}$.

Proof. Consider the subsheaf $\mathcal{F}_{C} \subset \mathcal{F} /\langle x y, z\rangle \mathcal{F}$ generated by boxes 四 $=(0,0,0) \in U_{a}$ whenever they exist. Then (the pre-image of) $\mathcal{F}_{C}$ must generate $\mathcal{O}_{C} \subset \mathcal{F}$. Hence, $\mathcal{F}$ has a subsheaf of the form $\mathcal{O}_{C}$ iff $\mathcal{F}_{C}$ is the direct sum of $\mathcal{O}_{E_{a b}}$ (with the trivial linearisation) where $E_{a b}:=E_{a} \cup E_{a+1} \cdots \cup E_{b}$ are connected components of supp $\mathcal{F}_{C}$. In other words, $\mathcal{F}_{C}$ is built from rods of degree $(\ldots,-\infty, 0,0, \ldots, 0,-\infty, \ldots)$; these are internal legs.

In the corresponding quasimap $\mathcal{V}$, the standard rods in $\mathcal{F}_{C}$ are specified by the hooks $\mathrm{hk}^{\square}$ for $\square=(0,0) \in \lambda_{a}$. Note that in (30), the leftward and rightward standard rods can attach to form $\mathcal{O}_{E}$ iff the maps $a$ and $b$ satisfy $[a: b]=[1: 1] \in \mathbb{P}^{1}$. This is also discussed in Example 14 . The generalisation to $\mathcal{A}_{m-1}$ is as follows. Let $\mathbb{V}_{C}[n]$ be the vector space at $\square=(0,0)$ in $\mathbb{V}[n]$, and consider the subflag

$$
\mathbb{V}_{C}[0] \subset \mathbb{V}_{C}[1] \subset \cdots \subset \mathbb{V}_{C}[\infty]
$$

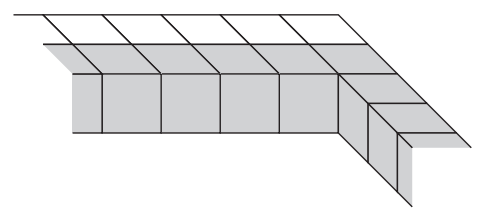

(a) Multiplication by $x_{b}$

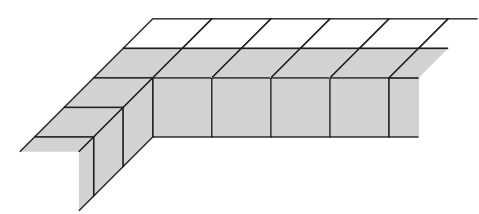

(b) Multiplication by $y_{b}$

Figure 13. Portions of adjacent local models. 
of the flag $\mathbb{V}^{\bullet} /\langle x, y\rangle \mathbb{V}^{\bullet}$. This is the quasimap analogue of $\mathcal{F}_{C}$. Then the leftward and rightward standard rods generated by boxes 四 $=(0,0,0)=U_{a}$ are attached to form $\mathcal{O}_{E_{a b}}$ iff this flag preserves the inclusion of the vector $(1,1, \ldots, 1) \in \mathbb{V}_{C}[\infty]$. But this is precisely the data of a framing $\mathcal{W} \hookrightarrow \mathcal{V}$.

If $\operatorname{dim} \mathbb{V}_{C}[\infty]=n$, then $\mathcal{F}$ has $n$ nontrivial (external) legs given by $n$ different hooks. The basic idea is that as we descend in the subflag $\mathbb{V}_{C}^{\bullet}$, standard rods in two hooks $\mathrm{hk}_{a}^{(0,0)}$ and $\mathrm{hk}_{b}^{(0,0)}$ 'glue' via a nontrivial step in the flag induced by

$$
\mathbb{C} \stackrel{1 \mapsto\left(\begin{array}{l}
1 \\
1
\end{array}\right)}{\longrightarrow} \mathbb{C}_{a} \oplus \mathbb{C}_{b}
$$

where $\mathbb{C}_{a}$ and $\mathbb{C}_{b}$ are the subspaces in $\mathbb{V}_{C}[\infty]$ corresponding to $\square=(0,0)$ in the two hooks. So $\operatorname{dim} \mathbb{V}_{C}[\infty]-\operatorname{dim} \mathbb{V}_{C}[0]$ is the number of exceptional curves $E_{a}$ in $\operatorname{supp} \mathcal{O}_{C}$.

4.3.4.

Although the equality of BS and quasimap vertices only requires matching stability conditions for T-fixed loci, it is in fact true that stability conditions match globally for the entire moduli spaces of quasimaps and $\pi$-stable pairs. The following proof was suggested by A. Okounkov.

Proposition 45. There is a T-equivariant isomorphism

$$
\pi \text {-Pairs } \text { nonsing } \infty_{\infty}\left(S \times \mathbb{P}^{1}\right) \simeq \text { QMaps }_{\text {nonsing } \infty}(\operatorname{Hilb}(S)) .
$$

Proof. Let $Y=S \times \mathbb{P}^{1}$ and $X=\mathbb{C}^{2} \times \mathbb{P}^{1}$. Consider the image

$$
\mathrm{P}:=\widetilde{\Psi}_{\mathrm{T}}(\pi \text {-Pairs } \text { nonsing } \infty(Y)) \subset D^{b} \operatorname{Coh}_{\mathrm{T}}(X) .
$$

The T-action on both moduli spaces means that they are unions of attracting subschemes for components of their T-fixed loci. Because $\widetilde{\Psi}_{\mathrm{T}}$ is an isomorphism on the T-fixed locus:

- P contains the T-fixed locus QMaps ${ }_{\text {nonsing } \infty}(\operatorname{Hilb}(S))^{\mathrm{T}}$;

$\circ$ by the upper semicontinuity of sheaf cohomology, elements of $P$ continue to be supported in a single cohomological degree away from $\mathrm{T}$-fixed loci as well, so $\mathrm{P} \subset \mathrm{Coh}_{\mathrm{T}}(X)$.

In addition, the equivalence $\Psi_{\mathrm{T}}$ sends sheaves supported on the exceptional divisor $E \subset \mathcal{A}_{m-1}$ to sheaves supported at $0 \in \mathbb{C}^{2}$, so fibres over $\mathbb{P}^{1}$ of elements in $\mathrm{P}$ are supported at $0 \in \mathbb{C}^{2}$. Therefore, elements of $P$ are (not necessarily stable) quasimaps, mapping at $\infty \in \mathbb{P}^{1}$ to the same point as for the original $\pi$-stable pair. But stability is an open and T-invariant condition, and therefore the destabilising locus is contained in the T-fixed locus. We already know that the T-fixed locus of $P$ is stable. Hence, $\mathrm{P} \subset \mathrm{QMaps}_{\text {nonsing } \infty}(\operatorname{Hilb}(S))$. An analogous argument for $\widetilde{\Phi}_{\mathrm{T}}$ shows it is an isomorphism.

\section{3D mirror symmetry}

\subsection{Via quasimap theory}

\subsection{1.}

The (classical and quantum) geometry of certain Nakajima quiver varieties $X$ is intimately related to the geometry of a mirror Nakajima quiver variety $\breve{X}$. In physics, this relation comes from $3 D$ mirror symmetry, also called symplectic duality, which is an S-duality between certain 3D $\mathcal{N}=4$ supersymmetric gauge theories. In our setting, these gauge theories are associated to a quiver and its 3D mirror, which can be constructed following [10, Section 3.3]. Specifically, affine type A quivers are 3D mirror to other affine type A quivers, but dimension vectors for their associated Nakajima quiver varieties are exchanged as in Figure 14. We say that the affine type A quiver is (conjecturally) self-mirror. 

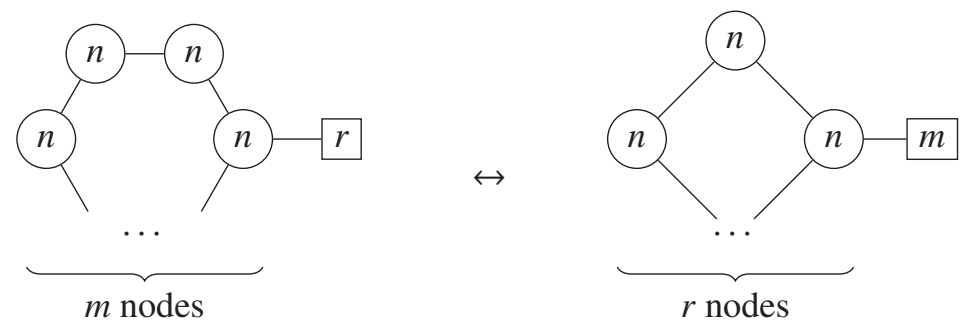

Figure 14. 3D mirror symmetry of affine type A quivers.

To identify the resulting Nakajima quiver varieties for the two chambers $C_{+}$and $C_{-}(m)$ that we are interested in, one can extend the isomorphisms of Theorems 19 and 20 to isomorphisms

$$
\begin{aligned}
\mathcal{M}_{\boldsymbol{\theta} \in C_{+}}\left(n \boldsymbol{v}^{0}, r \boldsymbol{w}^{0}\right) & \cong \mathcal{M}_{r}^{n}\left(\left[\mathbb{C}^{2} / \Gamma\right]\right) \\
\mathcal{M}_{\boldsymbol{\theta} \in C_{-}(m)}\left(n \boldsymbol{v}^{0}, r \boldsymbol{w}^{0}\right) & \cong \mathcal{M}_{r}^{n}(S)
\end{aligned}
$$

where $\mathcal{M}_{r}^{n}(S)$ is the moduli of rank-r instantons on $S$ of instanton number $n$. To be precise, an instanton on the surface $S$ is a torsion-free sheaf $\mathcal{E}$ on the compactified resolution $\bar{S} \rightarrow \mathbb{P}^{2} / \Gamma$ along with a choice of framing $\left.\mathcal{E}\right|_{\mathbb{P}} \cong \mathcal{O}_{\mathbb{P}}^{\oplus r}$ on the line at infinity $\mathbb{P} \subset \mathbb{P}^{2}$. The same applies to $\mathcal{M}_{r}^{n}\left(\left[\mathbb{C}^{2} / \Gamma\right]\right)$. Note that $\mathcal{M}_{1}^{n}(-)=\operatorname{Hilb}^{n}(-)$.

\subsection{2.}

We will now describe some standard mathematical expectations $(\star),(\star \star)$ and $(\star \star \star)$ for what 3D mirror symmetry entails (cf. [11, Section 1.6], [41, Definition 1.1]). These expectations were first explicitly described in [37].

Firstly, 3D mirror symmetry swaps the equivariant and Kähler variables of $X$ with those of $\check{X}$. More precisely, let $\mathrm{K}:=\operatorname{Pic}(X) \otimes_{\mathbb{Z}} \mathbb{C}^{\times}$, and let $\mathrm{T}=\mathrm{A} \times \mathbb{C}_{\hbar}^{\times}$act on $X$, where $\mathrm{A}$ is the subtorus preserving the symplectic form. Similarly, define $\check{\mathrm{K}}, \breve{\mathrm{T}}$ and $\breve{\mathrm{A}}$ for $\breve{X}$. Then the data of 3D mirror symmetry should include

( $\quad$ an isomorphism

$$
\kappa: \check{\mathrm{A}} \times \check{\mathrm{K}} \times \mathbb{C}_{\check{\hbar}}^{\times} \times \mathbb{C}_{q}^{\times} \stackrel{\sim}{\rightarrow} \mathrm{A} \times \mathrm{K} \times \mathbb{C}_{\hbar}^{\times} \times \mathbb{C}_{q}^{\times}
$$

that identifies $\breve{\mathrm{A}} \cong \mathrm{K}, \breve{\mathrm{K}} \cong \mathrm{A}$ and $\breve{\hbar}=q / \hbar$ and a bijection of fixed points

$$
b: X^{\mathrm{T}} \cong(\check{X})^{\check{\mathrm{T}}} \text {. }
$$

In the case of $X=\mathcal{M}_{r}\left(\mathcal{A}_{m-1}\right)$, our notation for the equivariant and Kähler variables will be as follows:

$\circ$ (Equivariant variables) Let $\mathrm{T}_{W} \subset G_{W}:=\prod \mathrm{GL}\left(W_{i}\right)$ be the framing torus, whenever the framing of the Nakajima quiver variety has dimension $>1$. Let $u_{1}, \ldots, u_{r}$ denote its weights. Then $\mathrm{T}:=\mathrm{T}^{\prime} \oplus \mathrm{T}_{W}$ where $\mathrm{T}^{\prime}$ is the usual torus with weights $x, y$ acting on $\mathcal{A}_{m-1}$ (from Subsection 2.1.2). Recall that $\hbar^{-1}=x y$, and let $t:=x / y$. Then the equivariant variables are the coordinates on $\mathrm{A}$ :

$$
t, \frac{u_{1}}{u_{2}}, \frac{u_{2}}{u_{3}}, \ldots, \frac{u_{r-1}}{u_{r}} .
$$

○ (Kähler variables) Recall that $\operatorname{Pic}(X)=\mathbb{Z} \mathcal{O}_{\text {Hilb }}(1) \oplus \operatorname{Pic}\left(\mathcal{A}_{m-1}\right)$. In this order, denote its generators by

$$
\mathfrak{z} \delta, \mathfrak{\jmath}_{1}, \ldots, \mathfrak{\jmath}_{m-1} \text {. }
$$

These are the Kähler variables. In the notation of Subsection 3.3.2, $\jmath_{\delta}=\jmath_{0} \jmath_{1} \cdots \jmath_{m-1}$. 
If $w$ is a variable for $X$, let $\breve{w}$ denote the analogous variable for $\check{X}$. Then the isomorphism $\kappa$ is given by

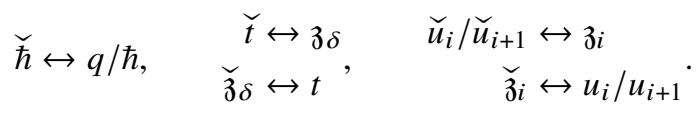

A fixed point in $X$ is an $m$-tuple of $r$-tuples of partitions $\lambda=\left(\lambda_{a, b}\right)_{\substack{0 \leq a<m \\ 0 \leq b<r}}$, which we view as an $m \times r$ matrix. Then the bijection $b$ of fixed points is the transpose map $\lambda \mapsto \lambda^{t}$.

5.1.3.

Let $\bar{\kappa}$ denote the isomorphism $\kappa$ restricted to $\breve{\mathrm{A}} \times \check{\mathrm{K}}$. Then $d \bar{\kappa}$ induces isomorphisms

$$
\begin{aligned}
\widetilde{\operatorname{Lie}} \mathrm{A} & \cong \operatorname{Pic}(X) \otimes_{\mathbb{Z}} \mathbb{C} \\
\widetilde{\operatorname{Pic}}(X) \otimes_{\mathbb{Z}} \mathbb{C} & \cong \text { Lie A. }
\end{aligned}
$$

For Nakajima quiver varieties, $\operatorname{Pic}(X) \otimes_{\mathbb{Z}} \mathbb{C}$ is exactly the (complexified) space of GIT stability conditions. A choice of stability chamber $C_{\text {eff }} \subset \operatorname{Pic}(X) \otimes_{\mathbb{Z}} \mathbb{C}$ identifies $X$ among all of its flops $\mathcal{M}_{\boldsymbol{\theta}}$ and is equivalent to the choice of effective cone. Let $\check{C}_{\text {eff }}$ be the effective cone of the mirror $\check{X}$. Given $X$ with a choice of attracting chamber $\mathfrak{C} \subset$ Lie A, 3D mirror symmetry should give

$(\star \star) \quad$ identifications $\check{C}_{\text {eff }} \leftrightarrow \mathfrak{C}$ and $\breve{\mathfrak{C}} \leftrightarrow C_{\text {eff }}$ via $d \bar{\kappa}$.

Example 46. For the stability condition $\boldsymbol{\theta}=(-m+1+\epsilon, 1,1, \ldots, 1)$ defining $\operatorname{Hilb}\left(\mathcal{A}_{m-1}\right)$, the corresponding point in Lie $\breve{\mathrm{A}}$ is given by $t=\sum \theta_{i}=\epsilon$ and $u_{i}-u_{j}=1$. (Here we conflate the K-theoretic weights $t, u_{i} \in \check{\mathrm{A}}$ with their logarithms in Lie $\breve{\mathrm{A}}$.) The chamber containing this point is therefore

$$
\check{\mathfrak{C}}_{-}:=\left\{u_{1} \gg u_{2} \gg \cdots \gg u_{m}>t>0\right\} .
$$

Similarly, the mirror chamber for $\operatorname{Hilb}\left(\left[\mathbb{C}^{2} / \Gamma\right]\right)$ is

$$
\check{\mathfrak{C}}_{+}:=\left\{t>u_{1} \gg u_{2} \gg \cdots \gg u_{m}>0\right\}
$$

\subsection{4.}

In an enumerative context, 3D mirror symmetry can be studied using quasimap theory following [1] and [37], using a key ingredient $S_{a b} b_{\mathfrak{C}}^{\text {EII }}$ called the elliptic stable envelope. Because the two geometries $X$ and $\breve{X}$ are mirror, one expects the quasimap vertices $\mathrm{V}_{\mathrm{QMaps}}(X)$ and $\mathrm{V}_{\mathrm{QMaps}}(\check{X})$ to be related. The relationship is best investigated using the $q$-difference equations ${ }^{5}$ that they satisfy in the equivariant and Kähler variables, first derived in [36, Section 8]. Let $\widetilde{V}_{\mathrm{QMaps}}$ be the normalisation of $\mathrm{V}_{\mathrm{QMaps}}$ so that these $q$-difference equations are scalar. Whatever the correct mathematical definition is for $X$ and $\breve{X}$ to be 3D mirror, we assume that it requires that

$$
(\star \star \star) \quad \widetilde{\mathrm{V}}_{\text {QMaps }}(X) \text { and } \widetilde{\mathrm{V}}_{\text {QMaps }}(\check{X}) \text { satisfy the same scalar } q \text {-difference equations, }
$$

up to the change of variables (33) which swaps the $q$-difference operators for equivariant and Kähler variables. Then $\widetilde{\mathrm{V}}(X)$ and $\widetilde{\mathrm{V}}(\breve{X})$ are distinguished among all solutions to the $q$-difference equations as the ones holomorphic in $\mathfrak{z}$ and $\breve{z}$, respectively, in a suitable neighbourhood of 0 in $C_{\text {eff }}$ and $\breve{C}_{\text {eff }}$. One can ask for the change of basis matrix transforming the basis of solutions holomorphic in $\mathfrak{z}$ to the basis holomorphic in $\breve{z}$. The main result of [1] is a geometric realisation of this matrix as a certain normalisation $\widetilde{\mathrm{Stab}_{\mathfrak{C}}}$ ofl the elliptic stable envelope.

${ }^{5}$ Here, the variable $q$ is identified with the weight of the torus acting on the quasimap domain $C$; we called this $z$ previously, in the context of the 3 -fold $Y=\mathcal{A}_{m-1} \times C$. 
Theorem 47 ([1, Theorem 5]). Suppose that $X$ is $3 D$ mirror to $(\check{X}, \check{\mathfrak{C}})$ in the sense that it satisfies $(\star)$, $(\star \star),(\star \star \star)$. Then

$$
\kappa^{*} \widetilde{V}_{\text {QMaps }}(X)=\widetilde{\operatorname{Stab}}_{\widetilde{C}}^{E l l} \widetilde{V}_{\text {QMaps }}(\check{X}),
$$

where $\kappa^{*}$ denotes the change of variables (33).

This theorem inspires the tentative mathematical definition of 3D mirror symmetry given in [41, Definition 1.1], where it is shown that the nonaffine type A quiver is self-mirror. Their definition should be equivalent to our three assumptions $(\star),(\star \star)$ and $(\star \star \star)$.

\subsection{5.}

The precise definition of $\widetilde{\mathrm{Stab}_{\mathfrak{C}}}$ Ell is unimportant for us, because we will mostly use Theorem 48 in the case where all equivariant variables of $X$ vanish. Namely, if $\sigma: \mathbb{C}^{\times} \rightarrow$ A is a generic cocharacter with $d \sigma \in \mathfrak{C}$, then we are interested in the limit

$$
\mathrm{V}_{\text {QMaps }}^{p}\left(\mathbf{0}_{\mathfrak{C}}, \boldsymbol{z}\right):=\lim _{w \rightarrow 0} \mathrm{~V}_{\text {QMaps }}^{p}(\boldsymbol{\sigma}(w), \mathfrak{z}) \in \mathbb{Z}(q, \hbar)\left[\left[\mathfrak{z}^{ \pm}\right]\right] .
$$

Equivalently, all Kähler variables of $\check{X}$ vanish, and $\widetilde{\mathrm{V}}_{\mathrm{QMaps}}^{p}(\check{X})$ is truncated to its constant term. The elliptic stable envelope becomes a diagonal matrix with very explicit entries. The overall effect is that (34) becomes very simple.

Corollary 48. For $p \in X^{T}$, let

$$
T_{p} X=T_{p}^{>0, \mathfrak{C}}+T_{p}^{<0, \mathfrak{C}}
$$

be a decomposition into attracting and repelling directions with respect to $\mathfrak{C}$. Then

$$
\kappa^{*} V_{Q M a p s}^{p}\left(\mathbf{0}_{\mathfrak{C}}, \boldsymbol{z}\right)=\prod_{w \in T_{b(p)}^{<0, \check{X} \check{X}}} \frac{\left(q \check{\hbar}^{-1} w^{-1} ; q\right)_{\infty}}{\left(w^{-1} ; q\right)_{\infty}},
$$

where $(w ; q)_{\infty}:=\prod_{n \geq 0}\left(1-q^{n} w\right)$ is the $q$-analogue of the Gamma function.

This relation between the quasimap vertex of $X$ and the $\breve{T}$-equivariant geometry of $\breve{X}$ was stated and checked in $[11,12]$ for cotangent bundles to flag varieties and $\mathrm{Hilb}\left(\left[\mathbb{C}^{2} / \Gamma\right]\right)$.

\subsection{6.}

The remainder of this article will explore the consequences of 3D mirror symmetry in the form of Corollary 48, between $X=\operatorname{Hilb}\left(\mathcal{A}_{m-1}\right)$ (and its flop $\left.\operatorname{Hilb}\left(\left[\mathbb{C}^{2} / \Gamma\right]\right)\right)$ and its mirror $\breve{X}=\mathcal{M}_{m}\left(\mathbb{C}^{2}\right)$. Specifically, via the BS/quasimaps correspondence, the formula (35) yields formulas for certain DT/PT/BS vertices of $Y=\mathcal{A}_{m-1} \times C$ that can be explicitly checked. Alternatively, the resulting statements for these vertices can be viewed as nontrivial evidence for the affine type A quiver being self-mirror.

\subsection{The Calabi-Yau limit}

\subsection{1.}

For equivariant K-theoretic objects on a 3-fold $Y$, setting $x y z=1$ is known as the Calabi-Yau (CY) limit. The evident self-duality of the tangent-obstruction theory (7) ensures that in this limit, the equivariant pushforward $\chi\left(M, \hat{\mathcal{O}}_{M}^{\text {vir }}\right)$ becomes the topological Euler characteristic $\chi(M)$ up to a sign (see [34, Section 3.1] for details). When $Y$ is a crepant resolution, it is known that

$$
\mathrm{V}_{\mathrm{CY}}^{\lambda}(Y):=\left.\mathrm{V}_{\pi \text {-Pairs }}^{\lambda}(Y)\right|_{x y z=1}=\left.\frac{\mathrm{V}_{\text {Pairs }}^{\lambda}(Y)}{\mathrm{V}_{\text {Pairs }_{\mathrm{exc}}}(Y)}\right|_{x y z=1}=\left.\frac{\mathrm{V}_{\mathrm{DT}}^{\lambda}(Y)}{\mathrm{V}_{\mathrm{DT}_{\mathrm{exc}}}(Y)}\right|_{x y z=1} \in \mathbb{Z}((Q))[[\boldsymbol{A}]]
$$

by [5] and [44] respectively; that is, the DT/PT/BS correspondences hold. 
It is possible to compute an explicit formula for $\mathrm{V}_{\mathrm{CY}}^{\lambda}(Y)$. Such an explicit formula is known in the physics literature under the guise of geometric engineering of (Nekrasov partition functions for) 4D $\mathcal{N}=2$ gauge theories with prescribed matter contents. Then we can verify (35) (in the CY limit) manually via the BS/quasimaps correspondence. Thus, the BS/quasimaps correspondence allows us to view this geometric engineering, in certain cases, as a consequence of $3 \mathrm{D}$ mirror symmetry.

\subsection{2.}

The CY limit $x y z=1$ becomes $q / \hbar=1$ in quasimaps language. Let $\mathrm{V}_{\mathrm{QMaps}, \mathrm{CY}}$ denote the quasimap vertex in the CY limit. On the mirror side, the CY limit becomes $\check{\hbar}=1$. Then there is massive cancelation on the right-hand side of (35), which yields (cf. [37])

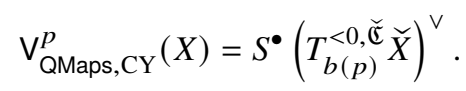

Here $S^{\bullet}$ can be viewed as the symmetric algebra functor on (virtual) vector spaces and is also known as the plethystic exponential. On $K_{\mathrm{T}}(\mathrm{pt})$, it can be defined as

$$
S^{\bullet}(w):=\frac{1}{1-w}, \quad S^{\bullet}\left(w+w^{\prime}\right)=S^{\bullet}(w) S^{\bullet}\left(w^{\prime}\right),
$$

where $w, w^{\prime} \in K_{\mathrm{T}}(\mathrm{pt})$ are monomials.

Proposition 49. Let $\check{\mathfrak{C}}=\left\{u_{1} \gg u_{2} \gg \cdots \gg u_{m} \gg t>0\right\}$. Then

$$
V_{C Y}^{\lambda}(Y)=\left.S^{\bullet}\left(T_{\lambda}^{<0, \check{\widetilde{C}}} \mathcal{M}_{m}\left(\mathbb{C}^{2}\right)\right)^{\vee}\right|_{(x, y)=\left(Q, Q^{-1}\right), u_{a} / u_{b}=A_{a b}} .
$$

This is exactly 3D mirror symmetry in the form of Corollary 48 for $X=\operatorname{Hilb}\left(\mathcal{A}_{m-1}\right)$ in the CY limit: Example 46 shows that $\breve{\mathfrak{C}}$ is mirror to $C_{\text {eff }}(X)$, and the change of variables is exactly the composition of $\kappa$ with the BS/quasimaps change of variables $Q \leftrightarrow \jmath_{\delta}$ and $A_{a b} \leftrightarrow z_{a b}$.

\subsection{3.}

The proof of Proposition 49 is by direct calculation. Whenever $Y$ is built from the local CY pieces $\operatorname{tot}(\mathcal{O} \oplus \mathcal{O}(-2))$ and $\operatorname{tot}(\mathcal{O}(-1) \oplus \mathcal{O}(-1))$, a convenient formalism for computing $\mathrm{V}_{\mathrm{CY}}(Y)$ is given in [20]. This certainly holds for $Y=\mathcal{A}_{m-1} \times \mathbb{C}$. These local CY pieces are special because it is possible to obtain a closed-form formula for their vertices, using the formula [38, Formula 3.23] for the topological vertex. Namely, let

$$
s_{\lambda}:=s_{\lambda}\left(Q^{-\rho}\right)=s_{\lambda}\left(Q^{1 / 2}, Q^{3 / 2}, Q^{5 / 2}, \ldots\right)
$$

be a principal $Q$-specialisation of the Schur function, and let

$$
[\lambda \mu]_{A}:=\prod_{i, j \geq 1} \frac{1}{1-A Q^{-\rho_{i}-\lambda_{i}} Q^{-\rho_{j}-\mu_{j}}}
$$

be the prefactor arising from applying (skew) Cauchy identities to certain (skew) Schur functions in the topological vertex. Then, using [20] or otherwise,

$$
\mathrm{V}_{\mathrm{CY}}^{\lambda}(Y)=Q^{\cdots} \frac{\prod_{a} s_{\lambda_{a}} \prod_{a<b}\left[\lambda_{a}^{t} \lambda_{b}\right]_{A_{a b}}}{\prod_{a<b}[\emptyset \emptyset]_{A_{a b}}},
$$

where $A_{a b}:=A_{a} A_{a+1} \cdots A_{b-1}$ for $a<b$, and $Q^{\cdots}$ denotes some monomial in $Q$ that is unimportant to us. 


\subsection{4.}

To begin relating $\mathrm{V}_{\mathrm{CY}}^{\lambda}(Y)$ to $T_{\lambda}^{<0} \mathcal{M}_{m}\left(\mathbb{C}^{2}\right)$, we need to introduce some notation for partitions. Recall that the arm-length and leg-length of a square $\square \in \lambda$ are

$$
\begin{aligned}
a_{\lambda}(\square) & :=\lambda_{i(\square)}-j(\square) \\
\ell_{\lambda}(\square) & :=\left(\lambda^{t}\right)_{j(\square)}-i(\square) .
\end{aligned}
$$

Let $n(\lambda):=\sum_{k}(k-1 / 2) \lambda_{k}$. Then a slight modification of Stanley's hook length formula yields

$$
s_{\lambda}=\frac{Q^{n(\lambda)}}{\prod_{\square \in \lambda}\left(1-Q^{a_{\lambda}(\square)+\ell_{\lambda}(\square)+1}\right)} .
$$

This formula for $s_{\lambda}$ can be written in terms of the geometry of Hilb $\left(\mathbb{C}^{2}\right)$ as follows. There is an explicit combinatorial formula

$$
\begin{aligned}
T_{\lambda} \operatorname{Hilb}\left(\mathbb{C}^{2}\right) & =\chi\left(\mathcal{O}_{\mathbb{C}^{2}}\right)-\chi\left(I_{\lambda}, I_{\lambda}\right) \\
& =\sum_{\square \in \lambda}\left(x^{-a_{\lambda}(\square)-1} y^{\ell_{\lambda}(\square)}+x^{a_{\lambda}(\square)} y^{-\ell_{\lambda}(\square)-1}\right)
\end{aligned}
$$

for the character of the tangent space at a fixed point $\lambda$. If $\check{\mathfrak{C}}=\{t>0\}$ is the choice of attracting chamber, then

$$
Q^{-n(\lambda)} s_{\lambda}=\left.S^{\bullet}\left(T_{\lambda}^{<0, \check{\mathscr{C}}} \operatorname{Hilb}\left(\mathbb{C}^{2}\right)\right)^{\vee}\right|_{(x, y)=\left(Q, Q^{-1}\right)} .
$$

\subsection{5.}

The generalisation of (39) to $\mathcal{M}_{m}\left(\mathbb{C}^{2}\right)$ is

$$
T_{\lambda} \mathcal{M}_{m}\left(\mathbb{C}^{2}\right)=\sum_{a, b} \frac{u_{b}}{u_{a}} T_{\lambda_{a}, \lambda_{b}},
$$

where $T_{\lambda, \mu}:=\chi\left(\mathcal{O}_{\mathbb{C}^{2}}\right)-\chi\left(I_{\lambda}, I_{\mu}\right)$. It is shown in the proof of [6, Lemma 6] that

$$
\begin{aligned}
T_{\lambda, \mu} & =\sum_{i, j \geq 1} x^{\mu_{i}-j} y^{-\lambda_{j}^{t}+i-1}-\sum_{i, j \geq 1} x^{-j} y^{i-1} \\
& =\sum_{\square \in \lambda} x^{-a_{\lambda}(\square)-1} y^{\ell_{\mu}(\square)}+\sum_{\square \in \mu} x^{a_{\mu}(\square)} y^{-\ell_{\lambda}(\square)-1} .
\end{aligned}
$$

It follows almost immediately that the remaining terms in (38) are

$$
\frac{\left[\lambda_{a} \lambda_{b}\right]_{A_{a b}}}{[\emptyset \emptyset]_{A_{a b}}}=\left.S^{\bullet}\left(A_{a b} T_{\lambda_{a}, \lambda_{b}}^{\vee}\right)\right|_{(x, y)=\left(Q, Q^{-1}\right)} .
$$

Proof (of Proposition 49). Note that $T_{\lambda} \operatorname{Hilb}\left(\mathbb{C}^{2}\right)=T_{\lambda, \lambda}$ and

$$
T^{<0, \check{\mathfrak{C}}} \mathcal{M}_{m}\left(\mathbb{C}^{2}\right)=\sum_{a} T_{\lambda_{a}, \lambda_{a}}^{<0, \check{\mathbb{E}}}+\sum_{a<b} \frac{u_{b}}{u_{a}} T_{\lambda_{a}, \lambda_{b}} .
$$

Hence, plugging (40) and (41) into (38) yields the desired equality up to a monomial $Q^{\cdots}$. This monomial must be trivial, because both sides are $1+O(Q, A)$. 


\subsection{6.}

It is worth mentioning that even without using the BS/quasimaps correspondence, 3D mirror symmetry in the CY limit for quasimap vertices already has many interesting consequences. For example, the degree configurations of T-fixed quasimaps to $\lambda \in \operatorname{Hilb}\left(\left[\mathbb{C}^{2} / \Gamma\right]\right)$ have been widely studied under the name of (coloured) reverse plane partitions (RPPs) of shape $\lambda$. These are ways to label the squares in $\lambda$ with nonnegative degrees $d_{\square}$ such that they are nondecreasing along rows and columns. If $\operatorname{deg}_{k}$ is the sum of $d_{\square}$ for all squares of colour $c(\square)=k$, then

$$
\mathrm{V}_{\mathrm{QMaps}, \mathrm{CY}}^{\lambda}\left(\operatorname{Hilb}\left(\left[\mathbb{C}^{2} / \Gamma\right]\right)\right)=\sum_{\pi \in \operatorname{RPP}(\lambda)} \boldsymbol{z}^{\operatorname{deg} \pi}
$$

is a generating series for coloured RPPs, because all fixed loci are isolated points. Then 3D mirror symmetry yields a closed-form formula for this series that matches with the known result in [14, Theorem 5.1]. More generally, [12] proves a formula for $\mathrm{V}_{\mathrm{QMaps}}^{\lambda}\left(\left.\mathrm{Hilb}\left(\left[\mathbb{C}^{2} / \Gamma\right]\right)\right|_{t=0}\right.$ to verify Corollary 48 in full generality, which can be viewed as a $(q, \hbar)$-deformation of that of [14].

A striking feature of these and related formulas is that they are plethystic, in the sense that the desired generating series can be written as $S^{\bullet}(\cdots)$. This is unsurprising via the CY limit of 3D mirror symmetry, which even explicitly identifies the terms in $\cdots$ using the equivariant geometry of the mirror.

\subsection{7.}

For $X=\operatorname{Hilb}\left(\mathcal{A}_{m-1}\right)$, the description of T-fixed quasimaps to $\lambda \in X^{\mathrm{T}}$ in Subsection 3.3.4 suggests to generalise RPPs as follows. For general $\lambda$, the components of $\mathrm{QMaps}^{\lambda}(X)$ are not isolated points, and it is not so clear what the enumerative significance of $\mathrm{V}_{\mathrm{QMaps}, \mathrm{CY}}^{\lambda}(X)$ is. But when $\lambda$ is empty except at a single $\lambda_{a} \neq \emptyset$, all fixed loci are isolated points. The resulting degree configuration is a generalised RPP $(G R P P)$ in the sense that each square is still labelled by a degree $d_{\square}$ and degrees are still nondecreasing along rows and columns, but

$\circ$ the underlying shape $V(\lambda)$ is a skew diagram with squares outside the positive quadrant in general;

$\circ$ the degrees labelling squares may be negative, with the condition that $d_{(0,0)} \geq 0$.

Then Proposition 49 can be viewed as a plethystic formula for the generating series of coloured GRPPs of shape $V(\lambda)$, which also admits a $(q, \hbar)$-deformation.

\subsection{Crepant resolution conjecture}

\subsection{1.}

Let $Y \rightarrow \mathfrak{Y}$ be a crepant resolution of a CY3 orbifold. One can study the orbifold DT/PT theory for $\mathfrak{Y}$ and obtain an orbifold topological vertex $\mathrm{V}_{\mathrm{CY}}^{\text {orb }}(\mathfrak{Y})$ [4]. When $\mathfrak{Y}$ satisfies the hard Lefschetz condition [13, Section 4], the DT crepant resolution conjecture (CRC) essentially asserts that

$$
\mathrm{V}_{\mathrm{CY}}(Y) \equiv \mathrm{V}_{\mathrm{CY}}^{\mathrm{orb}}(\mathfrak{Y}) \text {, }
$$

where we write $\equiv$ to emphasise that the equality is not as series but rather as rational functions. That both sides are indeed rational functions is not immediate; a proof is given in [2], which also proves the DT CRC, whenever $\mathfrak{Y}$ has projective coarse moduli space, for partition functions $Z_{\mathrm{CY}}(Y)_{\beta} \equiv Z_{\mathrm{CY}}^{\text {orb }}(\mathfrak{Y})_{\beta}$ enumerating curves of suitable classes $\beta$.

\subsection{2.}

For $Y=\mathcal{A}_{m-1} \times \mathbb{C}$ and $\mathfrak{Y}=\left[\mathbb{C}^{2} / \Gamma\right] \times \mathbb{C}$, the BS/quasimaps correspondence and 3D mirror symmetry yield a version of the DT CRC for fully equivariant and K-theoretic 1-leg vertices. In what follows, $\widetilde{\mathrm{V}}_{\text {Pairs }}(\mathfrak{Y})$ is exactly the (normalised) PT 1-leg vertex for $\mathbb{C}^{3}$, except with variables $\boldsymbol{Q}:=\left(Q_{0}, \ldots, Q_{m-1}\right)$ recording the number of boxes of each of the $m$ colours. 
Conjecture/Theorem 50 (Equivariant K-theoretic DT CRC). Using the bijection of fixed points of Lemma 24, identify $\operatorname{Coh}_{T}(Y)$ with $\operatorname{Coh}_{T}(\mathfrak{Y})$. There is an equality of rational functions

$$
\widetilde{V}_{\pi-P a i r s}(Y) \equiv \widetilde{R}_{\check{\mathbb{C}}_{-} \leftarrow \check{\mathfrak{C}}_{+}} \widetilde{V}_{\text {Pairs }}(\mathfrak{Y})
$$

where $\breve{\mathfrak{C}}_{ \pm}$are the attracting chambers of Example 46 and

$$
\widetilde{R}_{\mathbb{C}^{\prime} \leftarrow \mathfrak{C}}:=\widetilde{\operatorname{Stab}}_{\mathfrak{C}^{\prime}}^{E l l} \circ\left(\widetilde{\operatorname{Stab}}_{\mathfrak{C}}^{E I I}\right)^{-1}
$$

are (a certain normalisation of) elliptic R-matrices.

The conjectural aspect arises from whether $\operatorname{Hilb}\left(\mathcal{A}_{m-1}\right)$ is actually $3 \mathrm{D}$ mirror to $\mathcal{M}_{m}\left(\mathbb{C}^{2}\right)$ in the sense of $(\star \star \star)$, so that Theorem 48 is applicable. (Assumptions $(\star)$ and $(\star \star)$ are easily verified to hold.) In the CY limit this is verified by Proposition 49 and therefore what follows will constitute a proof. An alternate proof by direct computation was given in [42].

\subsection{3.}

We first explain how to reinterpret the DT CRC as a consequence of 3D mirror symmetry. Let

$$
\begin{aligned}
X_{-} & :=\operatorname{Hilb}\left(\mathcal{A}_{m-1}\right) \\
X_{+} & :=\operatorname{Hilb}\left(\left[\mathbb{C}^{2} / \Gamma\right]\right),
\end{aligned}
$$

which are flops of each other (across multiple walls). The BS/quasimap correspondence provides equalities

$$
\begin{aligned}
& \widetilde{\mathrm{V}}_{\text {QMaps }}\left(X_{-}\right)=\widetilde{\mathrm{V}}_{\pi \text {-Pairs }}\left(\mathcal{A}_{m-1} \times \mathbb{C}\right) \\
& \widetilde{\mathrm{V}}_{\text {QMaps }}\left(X_{+}\right)=\widetilde{\mathrm{V}}_{\text {Pairs }}\left(\left[\mathbb{C}^{2} / \Gamma\right] \times \mathbb{C}\right)
\end{aligned}
$$

after the appropriate changes of variables. Hence, the DT CRC is a comparison of $\mathrm{V}_{\mathrm{QMaps}}\left(X_{+}\right)$and $\mathrm{V}_{\mathrm{QMaps}}\left(X_{-}\right)$. On the 3D mirror side, Theorem 48 then yields

$$
\left(\widetilde{\operatorname{Stab}_{\widetilde{\mathbb{C}}_{+}}^{\text {EII }}}\right)^{-1} \widetilde{\mathrm{V}}_{\mathrm{QMaps}}\left(X_{+}\right)=\kappa^{*} \widetilde{\mathrm{V}}_{\mathrm{QMaps}}\left(\mathcal{M}_{m}\left(\mathbb{C}^{2}\right)\right)=\left(\widetilde{\operatorname{Stab}}_{\widetilde{\mathbb{C}}_{-}}^{\mathrm{Ell}_{-}}\right)^{-1} \widetilde{\mathrm{V}}_{\mathrm{QMaps}}\left(X_{-}\right),
$$

as desired. That all relevant 1-leg vertices are rational functions follows from more general expectations for fully equivariant quasimap vertices; see, for example, [43].

\subsection{4.}

In the CY limit, using (37), the DT CRC becomes a comparison of

$$
\mathrm{V}_{ \pm}:=\left.S^{\bullet}\left(T_{\lambda}^{<0, \check{\mathfrak{C}}_{ \pm}} \mathcal{M}_{m}\left(\mathbb{C}^{2}\right)\right)^{\vee}\right|_{(x, y)=\left(Q, Q^{-1}\right)}
$$

for the two different attracting chambers $\breve{\mathfrak{C}}_{ \pm}$of Example 46. Note that tangent spaces of symplectic spaces in general can be written as

$$
T_{\lambda}=T^{<0, \mathbb{C}}+\hbar\left(T^{>0, \mathfrak{C}}\right)^{\vee}
$$

Hence, a change of attracting chamber $\mathfrak{C} \leadsto \mathfrak{C}^{\prime}$ can only change $T^{<0}$ as

$$
T^{<0, \mathbb{C}^{\prime}}=T^{<0, \mathfrak{C}}-G+\hbar G^{\vee}
$$


for some subspace $G \subset T^{<0, \mathfrak{C}}$. The change of variables $(x, y)=\left(Q, Q^{-1}\right)$ implies $\hbar=1$. Using that

$$
S^{\bullet}(G)=(-1)^{\operatorname{dim} G} \operatorname{det}(G) S^{\bullet}\left(G^{\vee}\right),
$$

it follows immediately that

$$
\mathrm{V}_{+}=(\text {monomial }) \cdot \mathrm{V}_{-} .
$$

The discrepancy of an overall monomial factor in comparison to (42) comes from

$$
\widetilde{\mathrm{V}}_{\text {Pairs,CY}}(\mathfrak{Y})=\boldsymbol{Q}^{\cdots} \mathrm{V}_{\mathrm{CY}}^{\mathrm{orb}}(\mathfrak{Y})
$$

for some monomial $\boldsymbol{Q}^{\cdots}$ (see, e.g., [42, Theorem 3.1]). Presumably this monomial is exactly the CY limit of $\widetilde{R}_{\check{\mathfrak{C}}_{-} \leftarrow \check{\mathfrak{C}}_{+}}$.

Acknowledgements. This project sprouted from an (ongoing) attempt to prove a PT/quasimaps correspondence, to which I was first introduced by Noah Arbesfeld, Andrei Okounkov and Petr Pushkar. I would like to thank them along with Jim Bryan, Yakov Kononov and Andrey Smirnov for many productive discussions. I am especially grateful to Jim Bryan and Andrei Okounkov for reading a preliminary draft and for numerous helpful suggestions that improved the readability and content of this article.

Conflict of Interest: None.

\section{References}

[1] M. Aganagic and A. Okounkov, 'Elliptic stable envelopes', 2016, arXiv:1604.00423.

[2] S. V. Beentjes, J. Calabrese and J. Vold Rennemo, 'A proof of the Donaldson-Thomas crepant resolution conjecture', 2018, arXiv: 1810.06581 .

[3] T. Bridgeland, A. King and M. Reid, 'The McKay correspondence as an equivalence of derived categories', J. Amer. Math. Soc. 14(3) (2001), 535-554.

[4] J. Bryan, C. Cadman and B. Young, 'The orbifold topological vertex', Adv. Math. 229(1) (2012), 531-595.

[5] J. Bryan and D. Steinberg, 'Curve counting invariants for crepant resolutions', Trans. Amer. Math. Soc. 368(3) (2016), $1583-1619$.

[6] E. Carlsson and A. Okounkov, 'Exts and vertex operators', Duke Math. J. 161(9) (2012), 1797-1815.

[7] S. Cautis and T. Logvinenko, 'A derived approach to geometric McKay correspondence in dimension three', J. Reine Angew. Math. 636 (2009), 193-236.

[8] I. Ciocan-Fontanine and M. Kapranov, 'Virtual fundamental classes via dg-manifolds', Geom. Topol. 13(3) (2009), 17791804.

[9] I. Ciocan-Fontanine, B. Kim and D. Maulik, 'Stable quasimaps to GIT quotients', J. Geom. Phys. 75 (2014), 17-47.

[10] J. de Boer, K. Hori, H. Ooguri, Y. Oz and Z. Yin, 'Mirror symmetry in three-dimensional gauge theories, SL (2, ZZ)and D-brane moduli spaces', Nuclear Phys. B 493(1-2) 1997, 148-176.

[11] H. Dinkins and A. Smirnov, 'Characters of tangent spaces at torus fixed points and $3 d$-mirror symmetry', 2019, arXiv:1908.01199.

[12] H. Dinkins and A. Smirnov, 'Quasimaps to zero-dimensional $A_{\infty}$-quiver varieties', 2019, arXiv:1912.04834.

[13] J. Fernandez, 'Hodge structures for orbifold cohomology', Proc. Amer. Math. Soc. 134(9) (2006), 2511-2520.

[14] E. R. Gansner, 'The Hillman-Grassl correspondence and the enumeration of reverse plane partitions', J. Combin. Theory Ser. A 30(1) (1981), 71-89.

[15] V. Ginzburg, 'Lectures on Nakajima's quiver varieties', in Geometric Methods in Representation Theory. I, Vol. 24 of Sémin. Congr. (Soc. Math. France, Paris, 2012), 145-219.

[16] A. B. Givental, 'Equivariant Gromov-Witten invariants', Internat. Math. Res. Notices (13) (1996), 613-663.

[17] G. Gonzalez-Sprinberg and J.-L. Verdier, 'Construction géométrique de la correspondance de McKay', Ann. Sci. École Norm. Sup. (4) 16(3) (1984), 409-449.

[18] P. B. Gothen and A. D. King, 'Homological algebra of twisted quiver bundles', J. London Math. Soc. (2) 71(1) (2005), 85-99.

[19] K. Intriligator and N. Seiberg, 'Mirror symmetry in three-dimensional gauge theories', Phys. Lett. B 387(3) (1996), 513-519.

[20] A. Iqbal and A.-K. Kashani-Poor, 'The vertex on a strip', Adv. Theor. Math. Phys. 10(3) (2006), 317-343.

[21] S. Ishii, Introduction to Singularities (Springer, Tokyo, 2014).

[22] G. James and A. Kerber, The Representation Theory of the Symmetric Group, Vol. 16 of Encyclopedia of Mathematics and Its Applications (Addison-Wesley Publishing Co., Reading, MA, 1981). With a foreword by P. M. Cohn. With an introduction by Gilbert de B. Robinson.

[23] M. Kapranov and E. Vasserot, 'Kleinian singularities, derived categories and Hall algebras', Math. Ann. 316(3) (2000), $565-576$. 
[24] A. Kuznetsov, 'Quiver varieties and Hilbert schemes', Mosc. Math. J. 7(4) (2007), 673-697, 767.

[25] Y.-P. Lee, 'Quantum K-theory. I. Foundations', Duke Math. J. 121(3) (2004), 389-424.

[26] B. H. Lian, K. Liu and S.-T. Yau, 'Mirror principle. I', in Surveys in Differential Geometry: Differential Geometry Inspired by String Theory, Vol. 5 of Surv. Differ. Geom. ( International Press, Boston, MA, 1999), 405-454.

[27] D. Maulik, N. Nekrasov, A. Okounkov and R. Pandharipande', 'Gromov-Witten theory and Donaldson-Thomas theory. I', Compos. Math. 142(5) (2006), 1263-1285.

[28] D. Maulik, N. Nekrasov, A. Okounkov and R. Pandharipande', 'Gromov-Witten theory and Donaldson-Thomas theory. II', Compos. Math. 142(5) (2006), 1286-1304.

[29] D. Maulik, A. Oblomkov, A. Okounkov and R. Pandharipande', 'Gromov-Witten/Donaldson-Thomas correspondence for toric 3-folds', Invent. Math. 186(2) (2011), 435-479.

[30] K. Nagao', 'Quiver varieties and Frenkel-Kac construction', J. Algebra 321(12) (2009), 3764-3789.

[31] H. Nakajima, 'Instantons on ALE spaces, quiver varieties, and Kac-Moody algebras', Duke Math. J. 76(2) (1994), 365-416.

[32] H. Nakajima, Lectures on Hilbert Schemes of Points on Surfaces, Vol. 18 of University Lecture Series (American Mathematical Society, Providence, RI, 1999).

[33] H. Nakajima, 'Sheaves on ALE spaces and quiver varieties', Mosc. Math. J. 7(4) (2007), 699-722, 767.

[34] N. Nekrasov and A. Okounkov, 'Membranes and sheaves', Algebr. Geom. 3(3) (2016), 320-369.

[35] G. Oberdieck and A. Pixton, 'Holomorphic anomaly equations and the Igusa cusp form conjecture', Invent. Math. 213(2) (2018), 507-587.

[36] A. Okounkov, 'Lectures on K-theoretic computations in enumerative geometry', in Geometry of Moduli Spaces and Representation Theory, Vol. 24 of IAS/Park City Math. Ser. (American Mathematical Society, Providence, RI, 2017), 251-380.

[37] A. Okounkov, 'Enumerative symplectic duality', presented at MSRI workshop Structures in Enumerative Geometry, March 2018.

[38] A. Okounkov, N. Reshetikhin, and C. Vafa, 'Quantum Calabi-Yau and classical crystals', in The Unity of Mathematics, Vol. 244 of Progr. Math., (Birkhäuser, Boston, MA, 2006), 597-618.

[39] R. Pandharipande and R. P. Thomas, 'The 3-fold vertex via stable pairs', Geom. Topol. 13(4) (2009), $1835-1876$.

[40] R. Pandharipande and R. P. Thomas, 'Curve counting via stable pairs in the derived category', Invent. Math. 178(2) (2009), 407-447.

[41] R. Rimányi, A. Smirnov, A. Varchenko and Z. Zhou, 'Three-dimensional mirror self-symmetry of the cotangent bundle of the full flag variety', SIGMA Symmetry Integrability Geom. Methods Appl. 15 (2019), 22.

[42] D. Ross, 'Donaldson-Thomas theory and resolutions of toric A-singularities', Selecta Math. (N.S.) 23(1) (2017), 15-37.

[43] A. Smirnov, 'Rationality of capped descendent vertex in $K$-theory', 2016,arXiv:1612.01048.

[44] Y. Toda, 'Curve counting theories via stable objects I. DT/PT correspondence', J. Amer. Math. Soc. 23(4) (2010), $1119-1157$. 